\title{
Strategy Use of Children and Adults on Fraction Number Line Estimation
}

by

\section{Stephanie Hadden}

A thesis submitted to the Faculty of Graduate and Postdoctoral Affairs in partial fulfillment of the requirements for the degree of

Master of Arts

in

Psychology

Carleton University

Ottawa, Ontario

(C) 2020

Stephanie Hadden 


\begin{abstract}
The goal of this thesis is to compare adult and children strategy use on a fraction number line estimation task. Students in Grade 4 (64), Grade 6 (66) and university students (30) will complete whole number and fraction number line tasks as well as other measures of fraction skills and math achievement. Strategy reports were collected on the 0-1 fraction number line task for all groups, and adult strategy reports were also collected on the $0-5$ fraction number line task. Results showed that adults who used more strategies were more accurate on the $0-1$ fraction number line task. Moreover, when comparing performance across fractions on the $0-1$ fraction number line, adults performed similarly across all fractions whereas children were less accurate on the fractions in the middle of the number line. Implications of these findings for understanding the age-related differences in strategy use on a fraction number line are discussed. (150 Words)
\end{abstract}




\section{Acknowledgements}

First and foremost, I would like to thank my supervisor Dr. Jo-Anne LeFevre who has been so patient and encouraging throughout this process. She has challenged me to push myself throughout this thesis and I am so grateful to have had such a wonderful mentor for the past four years. Second, I would like to thank my loving family and friends who have supported me through very difficult times and have given me the strength to continue pursuing this degree no matter the obstacle. Third, a big thank you to my mentor, Heather Douglas, who not only provided some of the data for this research but was always available to answer any and all questions. Finally, to the members of my lab who have been a welcoming and resourceful community, I am so grateful for the knowledge you all have shared. 


\section{Table of Contents}

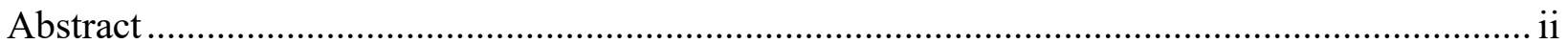

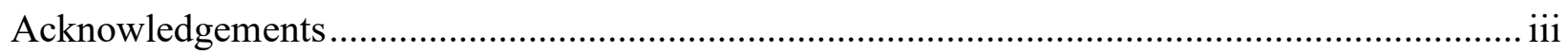

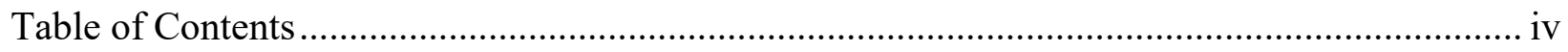

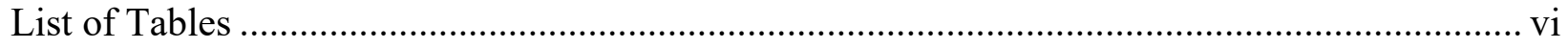

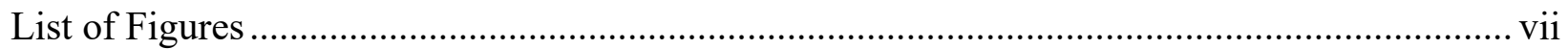

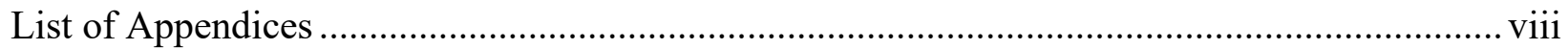

Strategy Use of Children and Adults on Fraction Number Line Estimation ................................... 1

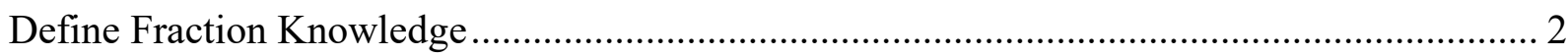

Importance of Fraction Knowledge ............................................................................ 2

Conceptual and Procedural Knowledge ........................................................................... 3

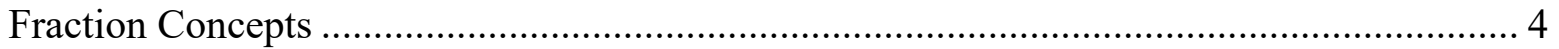

Fraction Learning in the Ontario Mathematics Curriculum ................................................. 5

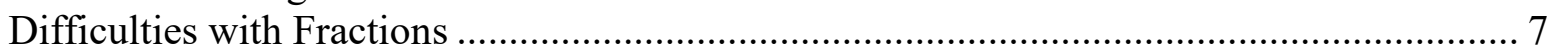

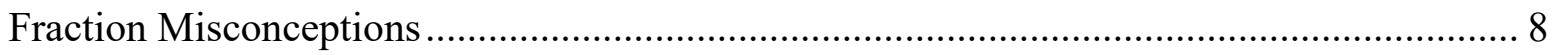

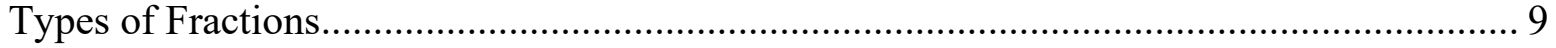

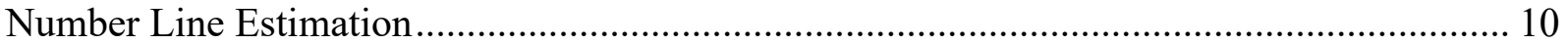

Integer Number Line versus Fraction Number Line ....................................................... 12

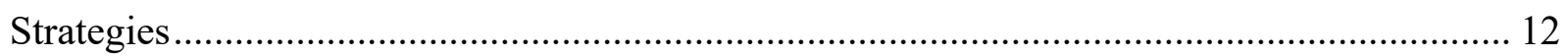

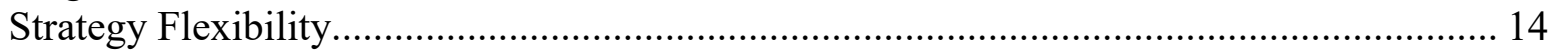

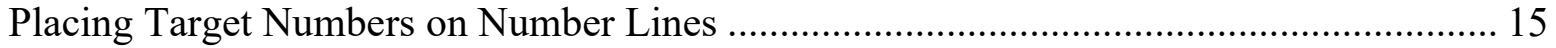

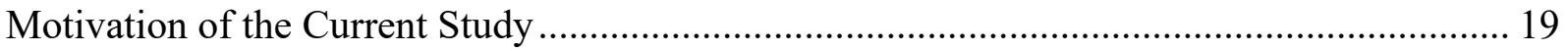

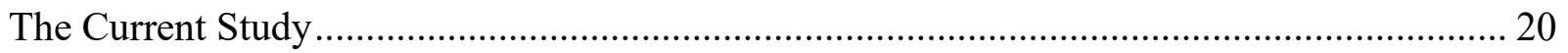

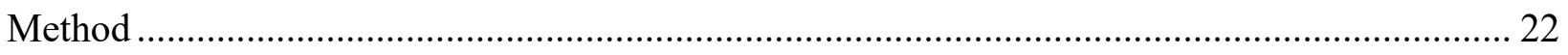

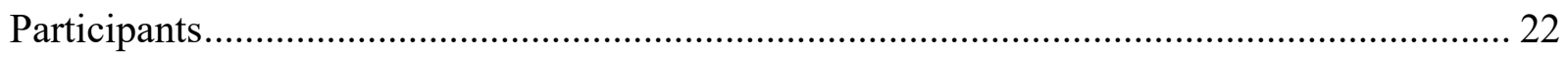

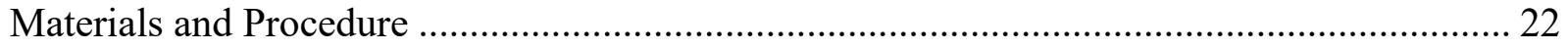

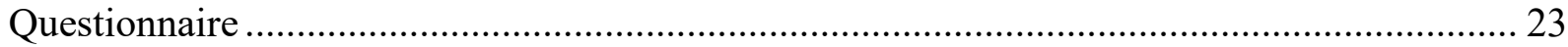

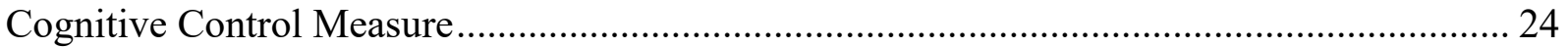

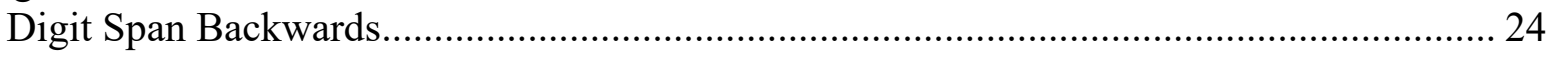

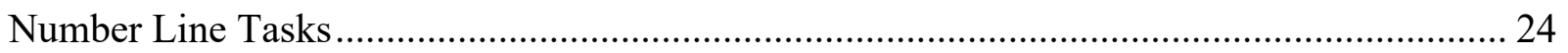

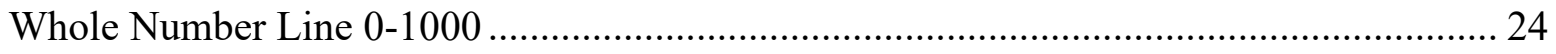

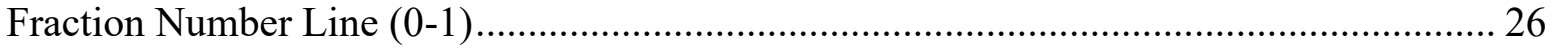

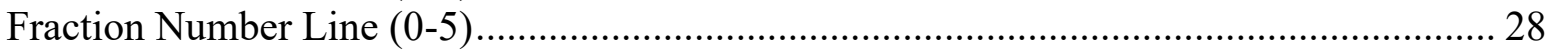

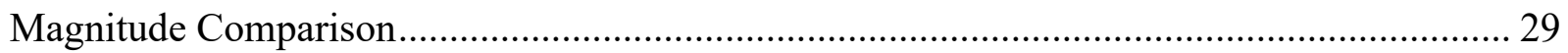

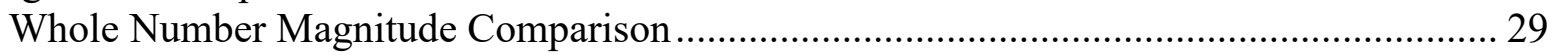

Fraction Magnitude Comparison ................................................................................. 30

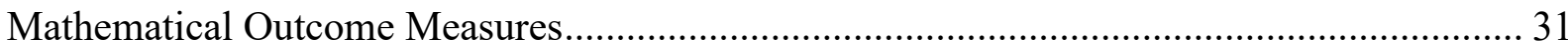

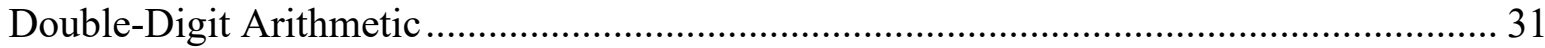




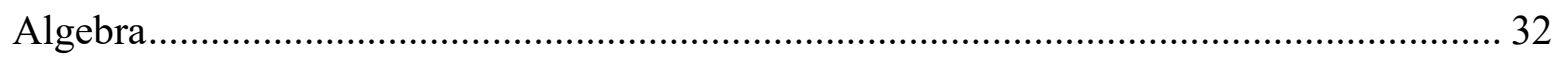

Fraction Knowledge Assessment .................................................................................... 33

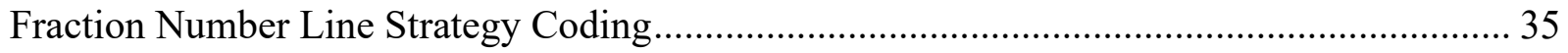

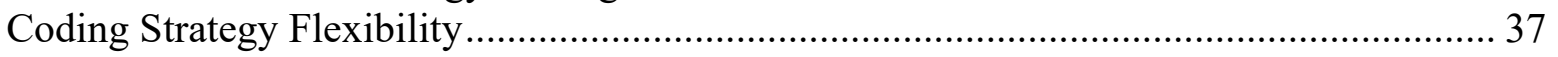

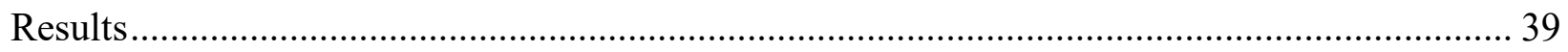

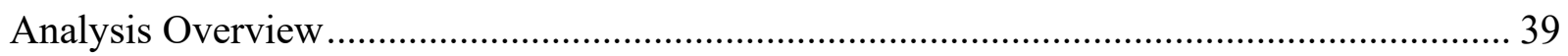

Effects of Grade

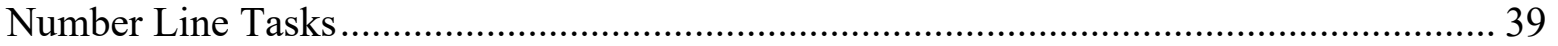

Grade Effects on Other Measures ................................................................................... 41

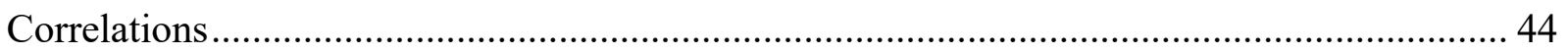

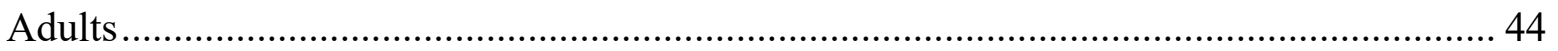

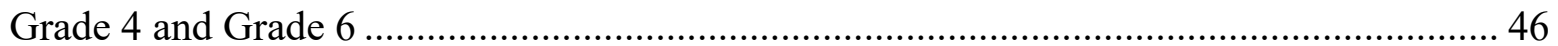

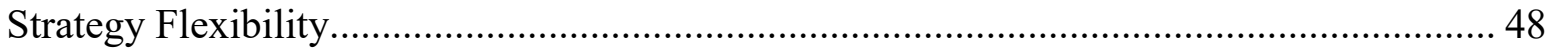

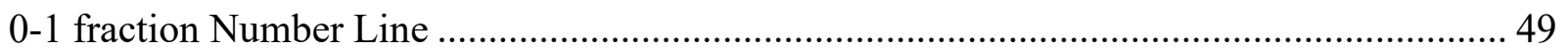

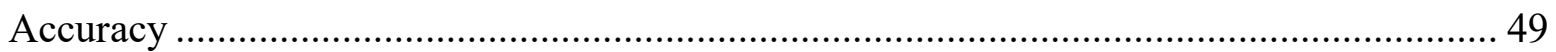

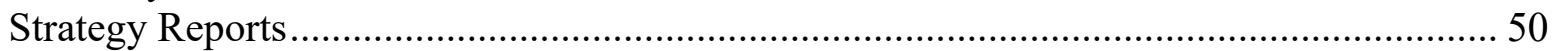

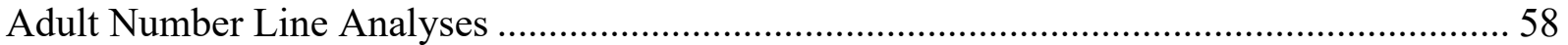

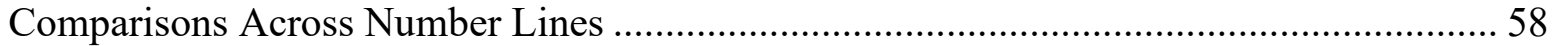

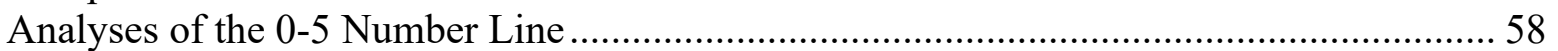

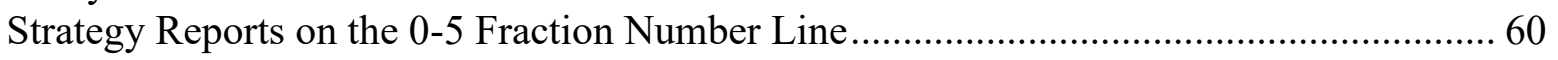

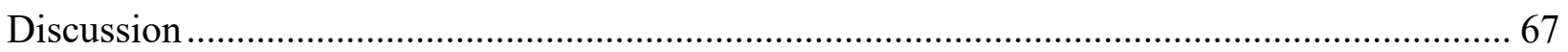

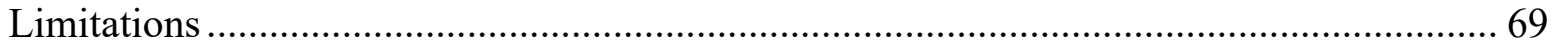

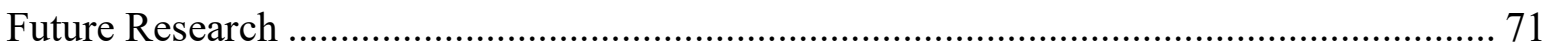

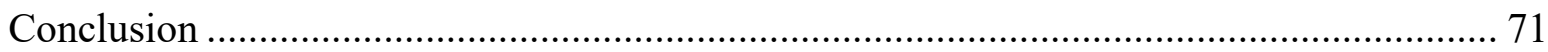

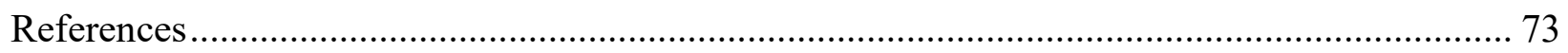

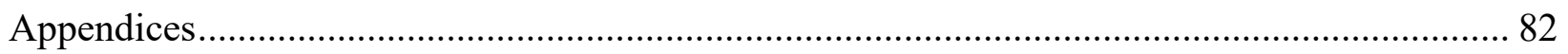




\section{List of Tables}

Table 1. List of Measures Completed by Each Age Group................................. 23

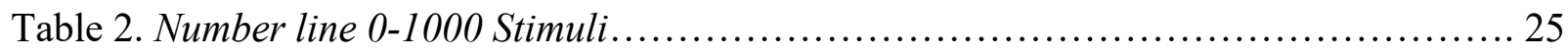

Table 3. Fraction Number Line 0-1 Stimuli......................................27

Table 4. Fraction Number Line 0-5 Stimuli........................................29

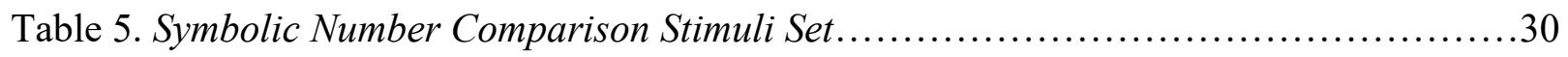

Table 6. Examples of Each Problem Type on the Fraction Magnitude Comparison Task........31

Table 7. Final Strategy Codes for the 0-1 Fraction Number Line.............................36

Table 8. Revised Final Strategy Codes for the 0-5 Fraction Number Line.......................37

Table 9. Descriptive Data for Number Line Tasks (Percentage Absolute Error)................40

Table 10. Descriptive Data for Cognitive, Numerical, and Fraction Tasks....................43

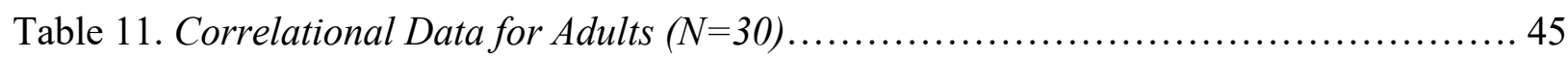

Table 12. Correlational Data for Grade 4 Children $(N=64)$ and Grade 6 Children $(N=66) \ldots . .47$ 


\section{List of Figures}

Figure 1. Fraction Number Line ......................................................... 2

Figure 2. Fraction Number Line Strategy Prompt................................... 26

Figure 3. Example of an item similar to those on the Key Math Algebra test.....................33

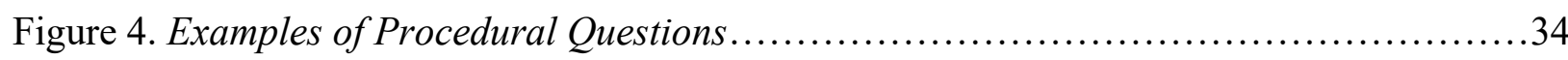

Figure 5. Examples of Conceptual Questions......................................... 34

Figure 6. Mean Percentage Absolute Error on Integer and Fraction Number Lines by Grade....41

Figure 7. Accuracy Collapsed across grade for each fraction on the 0-1 fraction number line.. 49

Figure 8. Accuracy by grade for placing the target numbers on the 0-1 fraction number line....50

Figure 9. Percentage of Students in Each Grade: Strategies for Placing $1 / 4 \ldots \ldots \ldots . . . \ldots . . . .52$

Figure 10. Percentage of Students in Each Grade: Strategies for Placing 3/7..................54

Figure 11. Percentage of Students in Each Grade: Strategies for Placing 4/5 .................55

Figure 12. Percentage of Students in Each Grade: Strategies for Placing 7/9..................56

Figure 13. Mean Percent Absolute Error by Fraction Format and Number of Digits in the

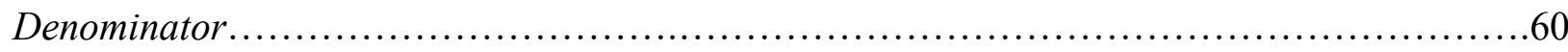

Figure 14. Accuracy for Placing Target Fractions .....................................6 61

Figure 15. Strategies Reported on the 0-5 Fraction Number Line for Improper Fractions.......62

Figure 16. Strategies Reported on the 0-5 Fraction Number Line for Mixed Numbers..........64 


\section{List of Appendices}

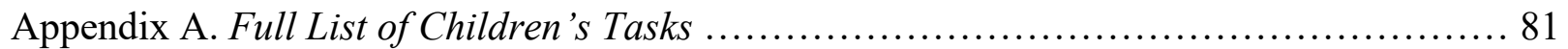

Appendix B. Full Adult Questionnaire.............................................. 82

Appendix C. Stimuli for Digit Span Backwards ........................................86

Appendix D. Full Fraction Knowledge Assessment. .....................................87 


\section{Strategy Use of Children and Adults on Fraction Number Line Estimation}

Rational numbers are numbers that can be expressed as the ratio of two integers. Thus, fractions (e.g., 3/4), decimals (e.g., 1.75) and percentages (e.g., 32\%) are all rational numbers. Children learn about fractions first before decimals or percentages, making fractions especially difficult. Misunderstanding fractions at this stage could derail children's learning about the other rational numbers. Thus, the early accumulation of fraction knowledge as students is relatively more important than other rational numbers. Throughout this thesis, I use the term fraction to refer to a rational number in fractional notation (i.e., $3 / 4,1 / 2,5 / 18$ ). Fractions are an integral part of the math curriculum. Knowledge of fractions is critical for achievement in more advanced areas of mathematics (Bailey, Hoard, Nugent, \& Geary, 2012; Rinne, Ye, \& Jordan, 2017; Siegler et al., 2012; Siegler, Thompson, \& Schneider, 2011). Because fractions are vital in progressing through mathematics education, it is important to understand how fraction knowledge is learned.

Fraction number lines (see Figure 1) can be an effective tool because their continuous nature may help children and adults better comprehend fractions (Siegler et. al., 2010). For example, the infinite nature and density of the rational number system is easily demonstrated with fractional number lines (Zhang et al., 2017). Fraction number line tasks are also useful for assessing fraction knowledge (Siegler et al., 2010; Kullberg, 2010; Noparit \& Saengpun, 2013). In the present research, I compared the fraction number line performance of students in grades 4 , 6 , and first-year university and asked participants to describe the strategies they used to make their estimates. No research has compared adults, who are relative experts, to children, who are relative novices, as they do the fraction number line task. In summary, the purpose of this thesis was to explore differences in fraction knowledge for students at a range of learning stages: 
Novice learners (grade 4), emerging learners (grade 6), and experienced users (first-year university students).

\section{Figure 1}

Fraction Number Line - in this iPad task, participants are shown a fraction in the center of the top of the screen and the instructions are: "touch the line where you think the fraction belongs".

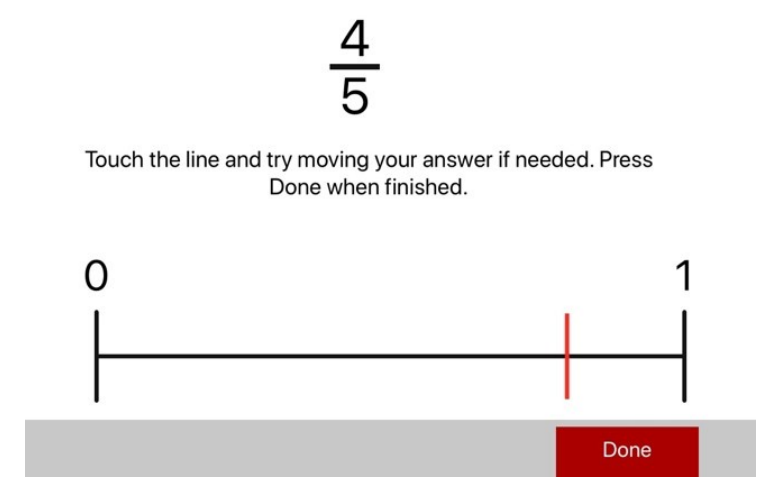

Below, I review the literature. First, I define fraction knowledge. Second, I review number lines and the research on both integer and fraction number line estimation. Third, I review the literature on strategy reports in math and how these analyses can be useful tools in understanding more about fraction knowledge and number line estimation. Finally, I will tie in fraction number lines and strategies, and review previous studies that have covered these areas.

\section{Define Fraction Knowledge}

\section{Importance of Fraction Knowledge}

Fraction knowledge is vital to students' mathematical learning and useful in everyday life. Students need to understand fractions before they can understand complex mathematics such as algebra, geometry, probability, and trigonometry. Equations in these areas are meaningless without previous knowledge of fractions. For example, to understand that the equation $1 / 6 \mathrm{X}=\mathrm{Y}$ means that $\mathrm{Y}$ is six times greater than $\mathrm{X}$, the solver must understand fraction operations. In a 
survey of teachers, a lack of knowledge of fractions and rational numbers was rated as the second biggest source of failure for students learning algebra (National Mathematics Advisory Panel, 2008), second only to solving word problems. Furthermore, mathematical proficiency and specifically fraction, ratio, and proportion knowledge are important for success in careers such as medicine, engineering, and software development (Davidson, 2012; McCloskey, 2007). Unfortunately, although fractions are an important consideration in mathematics education, everyday life, and future careers, they are very difficult to master.

\section{Conceptual and Procedural Knowledge}

Students need both procedural and conceptual knowledge to develop mathematical expertise (Rittle-Johnson, Siegler, \& Alibali, 2001). Procedural knowledge of fractions includes knowledge of the step-by-step procedures required to solve fraction arithmetic problems such as addition, subtraction, multiplication, and division. In contrast, conceptual knowledge involves the understanding of fraction properties, principles, and notation. For example, conceptual knowledge includes the understanding that fractions represent a part of an object or parts of objects, that fractions have magnitudes and can be ordered (i.e., $1 / 6,1 / 4,1 / 2$ ), and that fractions can be represented with symbolic notation (i.e., one third is represented by $1 / 3$ ).

An example of a problem that requires conceptual knowledge is shading in a circle to represent $1 / 2+1 / 4$. Solving this problem requires knowledge of the magnitude of each fraction, and that the shape should be divided into equal parts (i.e., part-whole knowledge). The procedural knowledge needed for the same problem includes two steps. First, find a common denominator. For $1 / 2+1 / 4$, the smallest common denominator is 4 as 4 is a multiple of 2 . Thus, change $1 / 2$ into the equivalent fraction $2 / 4$, by multiplying both the numerator and the denominator by 2 . Second, use the fraction addition procedure to add the numerators to produce 
the solution 3/4. People can memorize the procedural steps to solve fraction arithmetic problems without having conceptual understanding. However, students benefit from having conceptual knowledge above and beyond procedural knowledge because it allows speed and flexibility in problem solving (Crooks \& Alibali, 2014). For example, when students are asked to choose whether the sum of $13 / 14+11 / 12$ is closest to 1 or to 2 , their conceptual knowledge should help them to realize that the magnitude of both fractions is close to 1 and thus the sum is close to 2 . Conceptual knowledge allows a quick solution of the problem, whereas the procedural solution of finding common denominators would be computationally difficult.

In summary, conceptual knowledge is advantageous but is not needed for procedural problems where an algorithm can be implemented without understanding why it is implemented. However, conceptual knowledge of fractions accounts for variance in math achievement above and beyond fraction arithmetic skills (Siegler \& Pyke, 2012), whereas procedural knowledge does not account for variance above and beyond conceptual knowledge of fractions (Siegler et al., 2011). Hallett, Nunes and Bryant (2010) found individual differences in the way that students in grades 6 and 8 relied on conceptual versus procedural knowledge to solve problems; some relied more on procedures and some relied more on concepts. Thus, procedural and conceptual knowledge together allow people to be efficient and flexible in their strategy use when completing fraction problems or estimating (Zhang et al., 2017).

\section{Fraction Concepts}

In order for fraction knowledge as a whole to grow, children must be introduced to fractions in many different contexts (Hannula, 2003; Witherspoon, 2018). Fraction contexts include: part of a whole (1 of 2$)$, ratios (1:2), division (1/2), operator $(1 / 2(x+y))$, or measurement on a number line (1/2 located halfway between 0 and 1) (Behr \& Post, 1992). Across fraction 
contexts, there are similarities in conceptual knowledge such as understanding fraction magnitude, as well as knowing what the numerator and denominator represent. However, there are also differences that exist between fraction contexts such as the language surrounding fractions (e.g., For ratio: "one to two" vs. for division: "one divided by two"). Complete conceptual fraction knowledge requires mastery of fractions across all contexts. Although all fraction contexts are important, part-whole knowledge is the foundation of conceptual knowledge (i.e., children learn part of a whole first) in the early stages of fraction learning (Hannula, 2003).

Children's ability to make accurate fraction magnitude comparisons and judgements about relative quantities comes after the introduction of part-whole knowledge. If children understand that $7 / 8$ is a little less than 1 , then they should be able to reason that $7 / 8+7 / 8$ should be greater than 1 but less than 2 . However, understanding both fractions individually does not mean that children will understand the magnitude produced by the sum of the two fractions (Siegler \& Lortie-Forgues, 2015). In summary, no concept or context alone can successfully define fraction knowledge; only the convergence across fraction contexts can accurately demonstrate an individual's competency. Thus, no single task can capture a student's knowledge of fractions.

\section{Fraction Learning in the Ontario Mathematics Curriculum}

The mathematics curriculum for Ontario specifies the information children are expected to learn about fractions and when that information should be taught (Ontario, 2005). Children are to be exposed to fraction language from first grade. In Grades 1 and 2, students learn about equal sharing and teachers start to use fraction words (for example, "half of the class move to the right side of the room" or "share your cubes equally between the four members of your group"). 
However, symbolic fraction instruction (i.e., the use of symbolic fraction notation) does not start until grade 3. Up until this point, children have only been exposed to whole number symbols. In grade 3 , the Ontario curriculum goal is for children to develop an understanding of fractions as symbolic numbers (e.g., there are numbers between 0 and 1 , such as $3 / 4$ ) and to understand that these numbers can be placed on a number line.

The Grade 3 curriculum challenges students to know what the denominator means (e.g., for the fraction $1 / 2$, the denominator 2 signifies that the whole is divided into 2 equal parts). Moreover, in grade 3 students should learn about fraction equivalence (i.e., $3 / 6$ is the same as $1 / 2$ ) and then begin to reason about the relative size of fractions by making comparisons (i.e., $1 / 2$ is bigger than $1 / 4)$. Thus, children in Grade 3 are exposed to the beginnings of the important fraction concepts at their most basic level (e.g., with very simple fractions and whole number reference points $0-1)$.

Grades 4, 5, and 6 should prepare students for middle school and high school mathematics. In the Grade 4 Ontario curriculum, children are expected to compare and order simple fractions with emphasis on the benchmarks $0,1 / 2$, and 1 . The curriculum states that students should be able to understand fraction magnitudes and represent fractions in a variety of ways including traditional fraction notation. As well, children should be able to demonstrate knowledge of the numerator (e.g., for the fraction $2 / 3$, the numerator signifies that 2 parts of the whole are being referenced) and knowledge of equivalent fractions. In Grade 5, the Ontario Curriculum makes small steps in fraction knowledge; children should be able to make fraction comparisons with like denominators (including proper and improper fractions as well as mixed numbers) and relate fractions to decimals. Grade 6 requires students to compare and order fractions with unlike denominators and relate fractions to percentages. The curriculum states that 
students should be proficient at converting simple fractions to decimals and percentages. Thus, from grades 4 to 6 , children build on the same skills each year with more complex fractions and develop their conceptual knowledge.

If a student has a weak understanding of fractions moving into high school (i.e., beyond grade 8), they will experience a cascade of difficulties in mathematics. The next steps in the curriculum require knowledge of operations using fractions (grade 7) and the combination of fractions and whole numbers in algebraic equations (grade 8). The curriculum outlines increasingly difficult skills to master with fractions thus previous fraction knowledge provides the foundation for success on fraction tasks that require more complex procedural and conceptual knowledge.

\section{Difficulties with Fractions}

Many children in North America have a weak understanding of fraction concepts (LortieForgues, Tian, \& Siegler, 2015). There are many reasons that fractions are challenging. First, students build on their prior knowledge when they learn new concepts and so, when they start to learn about fractions, they refer to familiar whole number knowledge (Ni \& Zhou, 2005). This tendency is known as the whole number bias. For example, when comparing fractions, children incorrectly judge that $1 / 5$ is smaller than $1 / 10$ because the denominator 5 is less than 10 . Second, fractions have many meanings and, in English, are flexible in how they can be read (i.e., $3 / 4$ can be read as three out of four, three over four, three fourths, or three-quarters). This inconsistency in how fractions are discussed and interpreted in the classrooms and in everyday life leads to errors in understanding fractions across contexts (Hannula, 2003). Finally, fractions are presented in a unique format such that there is a combination of whole numbers and new symbols that make up one single fraction. Because children are used to dealing with whole 
numbers, they may have difficulty with this novel interpretation of seeing the combination of the numerator and denominator as a single number (Meert, Grégoire, \& Noël, 2008). These three challenges lead to misconceptions that may interfere with students' ability to interpret fractions.

\section{Fraction Misconceptions}

Misconceptions are errors in thinking. Misconceptions can happen at any and all levels of fraction knowledge. In the early stages of fraction learning, an example of a misconception is failing to understand that parts of the whole are of equal size (i.e., $2 / 3$ would represent 2 of 3 equal parts). With experience, misconceptions still exist such as not grasping the idea that there are an infinite number of fractions that refer to the same magnitude (Hansen et. al., 2015). Misconceptions lead to errors in problem solving which can lead to a lack of confidence when dealing with fractions. Moreover, when completing arithmetic with mixed numbers, students sometimes avoid the fractional parts of the operations as a result of their misconceptions (Fazio \& Siegler, 2011). Fraction misconceptions and resulting avoidance behaviours are common among students throughout schooling.

Fraction difficulties persist into adolescence and adulthood (Siegler \& Lortie-Forgues, 2015). Vamvakoussi and Vosniadou (2004) found that adolescents made mistakes when completing tasks that required the understanding that there are infinite rational numbers between two whole number endpoints. Adolescents (i.e., 12, 14, and 16 years old) failed to understand that there is no unique successor for each fraction like there is for integers. Moreover, in a large study of adolescents in the US, only $50 \%$ of $8^{\text {th }}$ graders correctly ordered the fractions $2 / 7,1 / 12$, and 5/9 from smallest to largest (Martin, Strutchens, \& Elliott, 2007). Adults also show evidence of having difficulty with fractions. In a survey that asked adults to choose the larger of two fractions (4/5 and 5/8), only $70 \%$ chose correctly where $50 \%$ is chance (Stigler, Givvin, \& 
Thompson, 2010). Furthermore, some adults used whole number knowledge incorrectly on fraction comparisons (DeWolf \& Vosniadou, 2015). Taken together, this evidence suggests that there is a constant challenge associated with distinguishing between whole number interpretations and the (correct) rational number interpretations of fractions throughout schooling and beyond.

\section{Types of Fractions}

To make fractions even more challenging, there are many different fraction types. Children learn proper fractions first and then over the years are exposed to the more complicated fraction types that require more advanced arithmetic. Proper fractions are fractions that are less than one (i.e., have numerators that are less than the denominators) (e.g., $3 / 4,1 / 2,5 / 13)$. Proper fractions can be reducible or non-reducible. Non-reducible fractions are fractions that cannot be reduced by dividing the numerator and the denominator by a common factor (e.g., $2 / 3,1 / 4,8 / 11$ ). In contrast, reducible fractions can be simplified by dividing the numerator and denominator by a common factor (e.g., $3 / 6$ can be reduced to $1 / 2$ ).

Fitzsimmons, Thompson, and Sidney (2020) tested the hypothesis that fractions with smaller components (i.e., non-reducible fractions) would be easier to place on a fraction number line than fractions with larger components (i.e., reducible fractions) with adults. They found that when comparing equivalent fractions adults were more precise in estimating non-reducible fractions than their reducible fraction counterparts. Adults also reported a higher level of familiarity with non-reducible fractions than their reducible fraction counterparts. This finding demonstrates that the type of fraction may be processed differently when estimating fraction magnitudes on a number line. 
What about fractions with the same magnitudes but represented in different formats? Improper fractions are fractions larger than one (i.e., have numerators that are greater than the denominators such as 14/5). Mixed numbers are whole numbers and proper fractions represented together (e.g., $24 / 5$ ). The procedure for transforming a mixed number to an improper fraction is (a) multiply the whole number by the denominator, (b) add the numerator to the product, and (c) write this product on top of the same denominator. For example, $24 / 5$ is converted to a mixed fraction by multiplying the whole number by the denominator of the fraction, that is, $2 \times 5=10$, adding this component to the numerator of the fractional component, that is, $10+4=14$, and then writing 14/5. These types of fractions may trigger different activations of processes for estimating fraction magnitudes on number lines.

\section{Number Line Estimation}

Number lines are tools that require numerical magnitude understanding (Namkung \& Fuchs, 2016). The use of a number line helps students make estimations and/or organize numbers because this is the representation that best aligns with the mental organization of numbers (i.e., increasing from left to right; Hamdan \& Gunderson, 2017). Placing numbers on a number line presumably reflects students understanding of the proportional relations between a symbolic number and the linear extent of the line. In a traditional number line task, a horizontal line is presented with labeled end points of zero 0 on the left and a base-ten number such as 100 or 1,000 on the right. Participants are presented with a target number that they must position on the number line. Number line estimation performance is correlated with math performance in adults and children (Schneider et al., 2018). Performance on fraction number line estimation is also correlated with math achievement (Hamdan \& Gunderson, 2017). However, compared to the large body of research on traditional number lines there is relatively less research on fraction 
number lines and the strategies used to place rational numbers on a number line (Zhang et al., 2017).

One view of the developmental progression of number line estimation is that children start with a logarithmic representation of numerical magnitude, that is, they exaggerate the distance between small numbers and minimize the distance between larger numbers (Feigenson et al., 2004). Over time and with education, this logarithmic representation changes to a linear representation where distance between consecutive integers is equal (Namkung \& Fuchs, 2016). From Grade 2, children start to be fairly accurate with their estimates and their number line estimates show linear representation of numerical magnitudes (Siegler \& Booth, 2008). Once this shift occurs, children are able to broaden their knowledge of magnitudes to include rational numbers, starting with fractions (Siegler et al., 2011). This change requires reorganization of number understanding to grasp the continuous nature of rational numbers (Siegler \& Pyke, 2013).

Another view suggests that developmental progression stems from the number line being important for proportional reasoning. Under this view, on the typical number line task, participants compare the target number to the bounded endpoint and make judgements about the numbers' relative magnitude. This viewpoint is modeled with a cyclical power function which demonstrates children's change from estimating using only the left endpoint (i.e., an open-ended judgement) to estimating with both endpoints (i.e., bounded judgement) (Slusser, Santiago, Barth, 2013). Similarly, to the logarithmic to linear shift model, fractions can be included once children can produce bounded judgements. 


\section{Integer Number Line versus Fraction Number Line}

A fundamental difference between fractions and integers that is salient in number line estimation is that fractions do not have predecessors and successors like integers do. Thus, when it comes to fraction number line estimation, there are infinite possibilities regardless of the endpoint. However, both integer and fraction number line estimation draw on some shared cognitive resources such as proportional reasoning (Namkung \& Fuchs, 2015). Moreover, training on integer and fraction number line estimation have demonstrated benefits in other math areas such as magnitude comparison (Hamdan \& Gunderson, 2017). Furthermore, both fractions and integers represent magnitudes (Fazio et al., 2014). Thus, do the fundamental differences between integers and fractions lead to differences in accuracy on number estimation tasks, or do the similarities between the tasks and their effects on math achievement lead to similar performance across integers and fractions?

Fazio et al. (2016) found that university students were as accurate at estimating fraction number line target fractions as estimating integer number line target numbers. However, adults and children with less expertise with fractions are less accurate at placing fractions on a number line than placing integers (Fazio et al., 2014, 2016; Siegler \& Opfer, 2003). Thus, based on existing research, both adults and children are less accurate at fraction number line estimation than integer number line estimation unless adults are high achieving in mathematics. If this is the case, perhaps strategy analyses could offer some insights on what makes an adult perform equally well on integer and fraction number lines.

\section{Strategies}

In order to fully capture fraction knowledge and development, we need to ask how and why each student arrives at their answer. Mistakes and thought process can allow researchers to 
see exactly what conceptual or procedural mistakes are being made, or which properties and principles are guiding students to the correct answer (Hannula, 2003). These in-depth analyses can also demonstrate whether participants have developed efficiency or flexibility in their approach (Fazio et al., 2016). It is important to look at well-researched mathematical tasks and understand more about what exactly these tasks are measuring and how the task demands might change for participants of different age groups. This method of strategy investigation can also be useful to inform future instruction (Zhang et al., 2017). Thus, analyzing strategies can allow for insights above and beyond outcomes.

Strategies are defined as "a procedure or set of procedures defined to achieve a higherlevel task or goal" (Lemaire \& Redder, 1999, p. 365). There are multiple ways of solving any problem. Both characteristics of the problem and characteristics of the learner influence strategy selection (Bonato, Fabbri, Umilta', \& Zorzi, 2007; Schneider \& Siegler, 2010). The same student can use different strategies for similar problems and different students can use similar strategies on the same problem. Ideally, students choose the most efficient strategy that they know to solve problems but this ideal is not always met. As students develop experience in a given area, students will develop preferences and rely on some strategies more than others (Fazio et al., 2016). These preferences can be both advantageous and problematic. Preference for a strategy might suggest that the student is confident in their ability to accurately execute the strategy consistently. However, it might also suggest that the student has only been able to memorize the procedure and has only limited conceptual knowledge of the problem. Thus, moving the focus away from outcomes and turning to the strategies that lead to behaviours is necessary. 


\section{Strategy Flexibility}

To demonstrate the importance of strategy analyses in research, I explore strategy use in other areas of math cognition. Areas in which researchers have used this approach to analyze individual differences are computations, and computational estimation. Insights about strategy use and flexibility from these areas can inform the present research.

Siegler (1989) examined strategy choice with respect to simple addition. Young children as early as preschoolers have been found to use multiple strategies to complete different computational problems. For example, young children chose between retrieval (retrieving an answer from memory) or a backup strategy (which involves some method to calculate the answer, e.g., counting from one or counting from the larger operand) to complete a problem. With simple addition, preschoolers used retrieval on easy problems that were familiar and used a backup strategy on more difficult problems that were less familiar. This flexibility allows children to fit the strategy to the demands of the problem to allow for optimal speed and accuracy.

Lemaire and Lecacheur (2002) investigated strategies on three-by-three-digit addition problems (e.g., $245 \times 162$ ) in adults, grade 6 , and grade 4 students. Participants were told to use one of two strategies: round up or round down. For the problem 245 x 162, the round-up strategy would lead to the problem $250 \times 170$ whereas the round-down strategy would lead to the problem $240 \times 160$. Participants in all groups favoured the round-down strategy over the roundup strategy. However, for problems that have operands with units smaller than 5, the round-down strategy was selected more often by the older groups. Younger students were less methodical in their strategy selection and thereby less influenced by problem features such as unit size. This study demonstrates that even when there are only two strategies available to the problem solver, 
being able to execute both strategies and know when to use both of them leads to improved accuracy and speed.

Xu, LeFevre, Wells, and Imbo (2014) explored strategic flexibility with computational estimation problems in two groups: Chinese-educated and Canadian-educated undergraduate students. Strategic flexibility was defined as choosing the optimal strategy that would lead to the most accurate estimation. When told to respond quickly, Chinese-educated participants were surprisingly less strategic than Canadian-educated participants. However, when the instructions were changed to encourage accuracy, Chinese-educated participants frequently chose the optimal strategy whereas Canadian-educated participants were much less likely to do so. This study demonstrates that strategic flexibility is important for accuracy and less important for speed.

In summary, these studies demonstrate that in other areas of mathematics, analyzing strategy reports has suggested that strategy flexibility is an important aspect of computation and estimation. Is using more strategies necessarily better? Or is accurately and quickly implementing the same strategy across problems just as effective? The present research aims to answer these questions across grades for fraction number line estimation.

\section{Placing Target Numbers on Number Lines}

How do students place target numbers on number lines? Children use a variety of strategies in number line estimation (Ashcraft \& Moore, 2012; Peeters, Degrande, Ebersbach, Verschaffel, \& Luwel, 2015). Young children use counting strategies to locate numbers on the number line (Xu \& LeFevre, 2016), often starting at the left endpoint and counting up. In contrast, older children tend to use benchmark strategies in combination with proportional reasoning to place their targets (Peeters, Sekeris, Verschaffel, \& Luwel, 2017). Di Lonardo, Huebner, Newman and LeFevre (2019) investigated adult strategy use on both a typical (0- 
$10,000)$ and an atypical (0-7000) number line using eye tracking. They found that adults used both endpoints and the midpoint as strategies on both types of number lines. Thus, adults and children alike use multiple strategies on integer number line estimation such as endpoints, midpoints, and other internal benchmarks.

For fractions, only three studies have explicitly included descriptions of strategy use on number lines (Sidney, Thalluri, Buerke, \& Thompson, 2019; Siegler, Thompson, \& Schneider, 2011; Zhang et al., 2017). Siegler, Thompson and Schneider (2011) assessed students in grade 6 and grade 8 on their ability to place fractions on $0-1$ and $0-5$ number lines. The authors categorized the strategies reported by the students into two main classes: transformation strategies (i.e., the original fraction was rounded to a whole number, transformed into a percentage, or changed to a mixed number) and segmentation strategies (i.e., using the denominator to divide the number line, or dividing based on the length of the line, that is, halves for $0-1$ and fifths for $0-5$ ). Results showed that grade 8 students used more transformation strategies than grade 6 students. Results also indicated that grade 8 students used more segmentation strategies than grade $6 \mathrm{~s}$ on the $0-5$ fraction number line but not on the $0-1$ fraction number line. Moreover, both grades performed better on the $0-1$ number line than the $0-5$ number line (Siegler et al., 2011). Thus, this study provides insight into how age might impact strategy use and how varying fraction number line lengths are more difficult for students.

Zhang et al. (2017) identified the same two classes of strategies (i.e., transformation and segmentation strategies) among students in grade 6 to 8 . However, they focused on faulty strategies and the errors that students made. They observed two common misconceptions that arise when students place fractions on a number line: ruler-tick-counting and misunderstanding the unit concept. Ruler-tick counting involves students confusing the ruler scale with the number 
line scale. In early education, before fractions are introduced children often use rulers for measurement, thus when children are tasked with number line estimation, they fall back on the ruler tick counting that they are familiar with. Misunderstanding the unit concept involves students identifying the right endpoint as one whole even if the number line is from 0-5.

Novillis-Larson (1980; cited by Behr 1983) also found misconceptions about the concept of unit for middle-schoolers but described only the errors, not the strategies. Novillis-Larson (1980) showed that students in Grade 7 struggled with using the unit of reference on the number line. On a 0-2 number line, students often made their estimates as though it was a 0-1 number line. With improper fractions, students used the end points as the "whole" and did not use one as the unit measurement. For example, on a 0-2 fraction number line when presented with 1/5 participants placed it as though the endpoint was 1 instead of identifying the magnitude of $1 / 5$ from 0-2. Mixed numbers, in contrast, were much easier to place on a number line because students could clearly see that the whole had to be more than one. Resnick et al. (2016) also found that students in grade 4 and 6 were better at positioning both proper fractions and mixed numbers than improper fractions on a 0-2 fraction number line. Overall, these findings provide more evidence that there are varying degrees of complexity between fraction number line lengths as well as fraction formats.

Hannula (2003) explored differences in fraction number line estimation performance as well as the misconceptions of students in grade 4 to grade 7 . Students were interviewed after they placed fractions on a number line. Some students incorrectly viewed $3 / 4$ as 3.4 while others were not yet able to identify $3 / 4$ as a number. Even in grade 7 , some students were not able to correctly identify the whole with simple fractions such as $3 / 4$. Comparing performance across grades showed that students in grade 7 performed much better than students in grade 5 on a 
fraction number line task and that students in grade 5 outperformed students in grade 4 on the same fraction number line task. This finding demonstrates the significant growth in fraction knowledge between grades 4 to 7 , however highlights the difficulties that remain even in grade 7.

Sidney et al. (2019) explored strategy flexibility on fraction magnitude comparison as well as fraction number line estimation in adults. However, their main goal was to link math anxiety, strategy variability and performance on fraction magnitude measures. The authors found that strategy variability was related to accuracy on both fraction tasks, however, this relationship was moderated by individual differences in working memory and mathematics background. They found that adults used a variety of strategies to make their estimates including transformation strategies, segmentation strategies, benchmarks, and a magnitude strategy (i.e., making reference to the fractions magnitude). Nevertheless, this research suggests that adults use a variety of strategies for fraction number line estimations and that this flexibility in strategy use is beneficial.

In addition to studies of strategy use on fraction number lines, there has also been research in the closely related area of strategy use for fraction magnitude comparisons. Fazio et al. (2016) found that university students from a highly selective university chose efficient strategies to make fraction magnitude comparisons and performed equally well on a $0-5$ fraction number line task and a 0-1000 number line task. In comparison, community college students did not choose efficient strategies and were much slower at making fraction magnitude comparisons than the university students. Community college students had fewer strategies to choose from and often did not select strategies on the basis of the specific problem presented. These students were also much more likely to use strategies that focused on separate parts of the fraction 
(numerator or denominator). These findings suggest that, even among adults, there may be substantial individual differences in fraction knowledge and strategy flexibility. Furthermore, these findings demonstrate that more strategies for comparing fraction magnitudes is advantageous. Similarly to fraction number line estimation, these results demonstrate that individuals who use more strategies show faster and more accurate performance than those who use fewer strategies.

\section{Motivation of the Current Study}

Previous studies on strategy reports in number line estimation have shown that adults and children alike use a wide range of strategies including, endpoint, midpoint, and other internal benchmark strategies. For fraction number lines, research has shown that older children use transformation and segmentation strategies to make their estimates (Siegler, Thompson, \& Schneider, 2011; Zhang et al., 2017). Research in the closely related area of fraction magnitude comparison highlights that strategy flexibility (using more strategies) are more accurate than those who use less strategies (Fazio et al., 2016; Sidney et al., 2019). Moreover, one study showed that using more strategies results in higher accuracy on fraction number line estimations with adults (Sidney et al., 2019). However, no research has shown the advantage of strategy flexibility for fraction number line estimation for children in comparison to adults. One possibility is that because the consistent use of some strategies allows participants to exhibit high accuracy across all trials (e.g., transforming fractions into a decimal), children might rely on one strategy. In contrast, the use of other strategies result in low accuracy across all trials (e.g., using only the denominator to make estimates), and thus relying on one strategy might be costly for accuracy. It may follow that the use of multiple strategies may not improve accuracy over using one reliable strategy. Despite this possibility, there are many strategies that involve reasoning 
about a fraction's magnitude that lead to accurate estimates (i.e., segmenting the line, transforming the fraction to a more familiar number, or relating the fraction to internal benchmarks). Therefore, thinking about a fraction's magnitude in different ways may help children and adults to make more accurate estimates, and may optimize speed in estimating.

\section{The Current Study}

In the present study, I compared children and adults' strategies on a fraction number line estimation task. Verbal strategy reports were collected. In order to capture fraction knowledge as a whole and situate it within the larger picture of math achievement, I also present participants' performance on conceptual and procedural fraction tasks, basic numerical tasks, and general math outcome measures. Participants were grade 4, grade 6, and first-year university students. These grades are targeted because in grade 4 , fractions start to become a focus in the math curriculum and by grade 6 , students should have foundational fraction knowledge. These time points are critical in a student's development of fraction knowledge. The third group was made up of first-year undergraduate students with a median age of 18 . These students had finished high school and are expected to be more advanced in their fraction knowledge than grade 6 students because of the additional six years of schooling. Thus, these adults should be experts relative to the children.

Based on the assumption that expertise varies with experience, I predicted that:

1) Adults will perform better on all the tasks than both groups of children.

2) Grade 6 children will perform better on all the tasks than grade 4 students. With respect to strategy flexibility:

3) Strategy flexibility will be related to the mathematical outcome measures for all three groups. 
4) Adults will use more strategies than children on the 0-1 fraction number line.

5) Children in grade 6 will be more variable in their strategy use on the $0-1$ fraction number line than children in grade 4.

Adults also completed a 0-5 fraction number line task as well as the other measures completed by children. The stimuli included both improper and mixed fractions, with either single- or double-digit denominators (e.g., 20/9, 37/14, 3 2/5, 3 13/18).

6) Adults will be less precise in their estimates on the 0-5 fraction number line as they will be on the $0-1$ fraction number line.

7) Adults will be less precise when estimating improper fractions than proper fractions or mixed numbers on the 0-5 fraction number line.

8) Adults will be less precise when estimating for fractions with single- than with double-digit denominators. 


\section{Method}

\section{Participants}

Students were recruited from the Upper Canada District School Board as part of a larger study on language learning and math achievement. The sample included students in grade $4(N=$ $64,34$ boys $)$ and in grade $6(N=66,38$ boys). The grade 4 children had a mean age of 113 months (9:5 years:months) and the grade 6 children had a mean age of 137 months (i.e., 11:5 years:months). All of the children spoke English as their first language.

My goal was to recruit 60 undergraduate students. However, due to COVID-19 I had to stop collecting data after 30 participants. Thus, undergraduate students $(N=30,14$ men) with a median age of 18 years (range 18 - 22) were recruited from first-year psychology and cognitive science classes. These students received course credit for their participation. Participants were from diverse disciplines, variously pursuing Bachelor of Arts degrees (40\%), Bachelor of Computer Science degrees (16.7\%), Bachelor of Science degrees (13.3\%), Bachelor of Communications (13.3\%), Bachelor of Engineering (10\%), or other degrees (6.7\%).

\section{Materials and Procedure}

Children completed a range of tasks in three 30-min sessions. These tasks included measures of cognitive skills, math performance, fraction assessments, and number line estimation tasks. For the full list of the tasks and procedure please see Appendix A.

Adults completed 10 of the tasks that children were tested on in one 40-minute session. Four of these tasks were identical to the versions that children were tested on $(0-1000$ number line, 0-1 fraction number line, whole number magnitude comparison, and digit backwards). Four of the tasks were augmented with additional questions or more difficult versions were created (i.e., questionnaire, fraction knowledge assessment, key math algebra, arithmetic fluency). 
Adults completed two additional tasks: a 0-5 fraction number line task and a fraction magnitude comparison task. Table 1 outlines the tasks, times, and the age group that completed each task.

\section{Table 1}

List of Measures Completed by Each Age Group.

\begin{tabular}{clcccc}
\hline Order & Task & Time & Grade 4 & Grade 6 & Adults \\
\hline 1 & Questionnaire & 2 & $\checkmark$ & $\checkmark$ & $\checkmark+$ \\
2 & Fraction Number Line 0-1 & 3 & $\checkmark$ & $\checkmark$ & $\checkmark$ \\
3 & Fraction Number Line 0-5 & 3 & & & $\checkmark$ \\
4 & Fraction Knowledge Assessment & 10 & $\checkmark$ & $\checkmark$ & $\checkmark+$ \\
5 & Fraction Magnitude Comparison & 3 & & & $\checkmark$ \\
6 & Key Math Algebra & 7 & $\checkmark$ & $\checkmark$ & $\checkmark+$ \\
7 & Digit Backwards & 3 & $\checkmark$ & $\checkmark$ & $\checkmark$ \\
8 & Whole Number Comparison & 3 & $\checkmark$ & $\checkmark$ & $\checkmark$ \\
9 & Arithmetic Fluency & 1 & $\checkmark$ & $\checkmark$ & $\checkmark+$ \\
10 & Number Line 0-1000 & 3 & $\checkmark$ & $\checkmark$ & $\checkmark$ \\
\hline
\end{tabular}

Note: +: More advanced questions were added to challenge the older participants.

\section{Questionnaire}

For the children in Grades 4 and 6, teachers sent consent forms home with the students. If parents wanted their child to participate, they signed the consent form and sent it back to the school. Along with the consent form was a page of questions such as parents' highest level of education, child's age/sex, child's first language, whether the child spoke other languages, and how often the child spoke each language. In contrast, undergraduate students were presented with the consent form in person. Consent forms were given, at the beginning of the test session, on an iPad with the same questions from the children's survey and additional questions such as: 
when was your last math course, what program are you in, and attitudes towards fractions and math overall. For the full list of questions, see Appendix B.

\section{Cognitive Control Measure}

\section{Digit Span Backwards}

In this task, participants listened to a set of numbers presented via audio files on an iPad application. Once they heard a beep, which meant that all the numbers for that trial were presented, participants had to repeat the numbers back to the experimenter in reverse order. The instructions were as followed: "I am going to say some numbers. Listen carefully and when I am through, say them backwards. Let's practice! If I say 3, 1, what would you say? Can you say those two numbers backwards? 1, 3.". Participants received two practice trials with 2-digits to ensure that they understood the task. Each of the following spans had two trials from a 2-digit span to 8-digit span. Testing was discontinued if participants answered incorrectly for both trials in a given span. Scoring was the total number of correct responses. Reliability between trials 1 and trials 2 across spans was low (Cronbach's alpha $=.452)$. See Appendix C for the full list of stimuli.

\section{Number Line Tasks}

Students' accuracy on the number line estimation tasks was measured by percent absolute error (PAE), as shown in Equation (1). For example, if the target number was $1 / 4$ and the estimate was placed at $1 / 2$, PAE would be $(|1 / 2-1 / 4| / 1.0) \times 100=25 \%$.

$\mathrm{PAE}=(\mid$ Estimate - Target Number $\mid /$ Scale of Number Line $) \times 100$

\section{Whole Number Line 0-1000}

In this iPad task (Hume \& Hume, 2014), participants see a number line that ranges from 0-1000. A target whole number from 0-1000 is presented in the center of the top of the screen. 
Participants must tap the number line where they think the number is located from 0-1000. For children, before the test begins the instructions are as followed: "We call this black line 'a number line' because there are numbers on it. At one end ("pointing to ' 0 '), we have number 0 ; at the other end ('pointing to '1000'), we have number 1000. There are some other numbers hidden in the line, and your job is to find those numbers one by one." Then, during practice, children receive added instruction: “"“Can you tap a location on this line where you think number 100 belongs?" "Good. Let's try another number”.

For undergraduates, the instructions are simplified: "This is a number line from 0 (point) to 1000 (point). A number will appear at the top of the screen. Your job is to tap the number line where you think that number belongs. Any questions?” There is a total of 30 trials. Reliability across trials was high (Cronbach's alpha $=.937)$. For the complete list of stimuli see Table 2.

\section{Table 2}

Number Line 0-1000 Stimuli

\begin{tabular}{ccccc}
\hline \multicolumn{6}{c}{ Sections of the Number Line } \\
\hline $0-200$ & $200-400$ & $400-600$ & $600-800$ & $800-1000$ \\
\hline 6 & 211 & 420 & 617 & 835 \\
18 & 239 & 458 & 656 & 876 \\
59 & 344 & 542 & 761 & 903 \\
97 & 383 & 580 & 789 & 941 \\
124 & & & 982 \\
165 & & & 994 \\
\hline
\end{tabular}




\section{Fraction Number Line (0-1)}

In this iPad task, participants are presented with a number line with end points 0 and $1 . \mathrm{A}$ target fraction appears in the center of the top of the screen and participants are told to place their finger on the number line where they think the fraction belongs (see Figure 2). The stimuli were all non-reducible proper fractions with single-digit denominators (see Table 3 for the list of stimuli).

Figure 2

Fraction Number Line Strategy Prompt

$$
\frac{4}{5}
$$

Touch the line and try moving your answer if needed. Press

Done when finished.

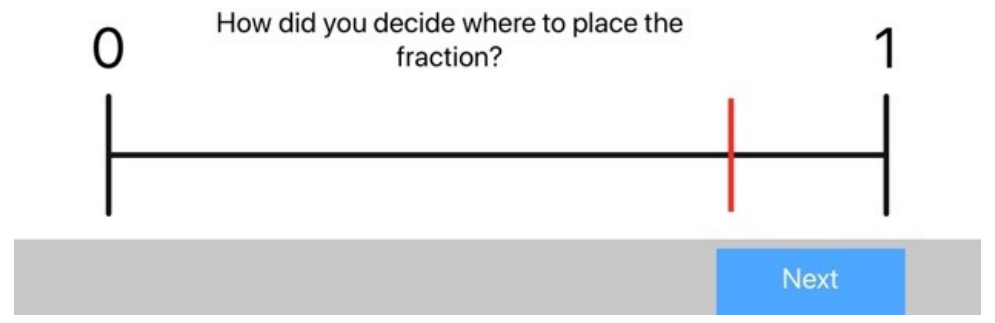




\section{Table 3}

Fraction Number Line 0-1 Stimuli

\begin{tabular}{cccccccc}
\hline \multicolumn{7}{c}{ Denominator } \\
\hline 2 & 3 & 4 & 5 & 6 & 7 & 8 & 9 \\
\hline $1 / 2$ & $1 / 3$ & $1 / 4^{*}$ & $1 / 5$ & $1 / 6$ & $1 / 7$ & $1 / 8$ & $1 / 9$ \\
& $2 / 3$ & $3 / 4$ & $2 / 5$ & $5 / 6$ & $2 / 7$ & $5 / 8$ & $2 / 9$ \\
& & $3 / 5$ & & $3 / 7 *$ & $7 / 8$ & $4 / 9$ \\
& & $4 / 5^{*}$ & & $4 / 7$ & & $5 / 9$ \\
& & & $5 / 7$ & & $7 / 9 *$ \\
& & & $6 / 7$ & & $8 / 9$ \\
\end{tabular}

* For these fractions, students were prompted to explain their strategy use.

On the first four trials, participants were asked about the strategies they used after placing the fractions. They were prompted with "How did you decide to place the fraction there?" and answered to the best of their ability (see Figure 2). The experimenter transcribed their response as well as classified their strategy. The prompted fractions were: $1 / 4,4 / 5,7 / 9$, and 3/7. These fractions were selected because they closely align with one of the strategies that I expected to see children and adults use. The fraction $1 / 4$ allows for the number line to be easily divided into four equal parts. The fraction $4 / 5$ can be converted to $80 \%$ and thus some students might use a transformation strategy to place this fraction. The fraction $3 / 7$ is close to $1 / 2$ therefore students might use a reference point to make this estimate. Finally, the fraction 7/9 does not fit neatly into any of these categories and so we were interested in what a student might do when presented with this fraction. On the remaining 26 trials, participants positioned fractions without indicating their strategies. Scoring for this task was percent absolute error see Equation 1. Reliability across trials was high for percentage absolute error (Cronbach's alpha $=.884$ ). 


\section{Fraction Number Line (0-5)}

Only adults completed this task. The procedure is identical to the $0-1$ number line task except that the high endpoint was 5 and there were more stimuli. The set included improper fractions and mixed numbers to capture the full range of the number line. There were eight fractions prompted for strategy use: one mixed number and one improper fraction from each section of the number line (except $0-1$ ). The prompted fractions were: 9/5, $19 / 16,32 / 15,27 / 8$, 41/11, 3 5/7, 92/19, 4 4/9.

The set of fractions were selected based on where they were on the number line. An equal number of fractions were selected from each section of the number line, including the midpoint. The first section (0-1) is comprised of only 6 proper fractions, half of which had double digit denominators (11-19). All other sections had six mixed numbers and six improper fractions (balanced for \# of digits in the denominator). Mixed numbers and improper fractions were paired and equal in magnitude. Reliability across trials was moderate (Cronbach's alpha $=.638$ ). For the complete list of stimuli, see Table 4. 


\section{Table 4}

Fraction Number Line 0-5 Stimuli

\begin{tabular}{|c|c|c|c|c|}
\hline \multicolumn{5}{|c|}{ Sections of the Number Line } \\
\hline $0-1$ & $1-2$ & $2-3$ & $3-4$ & $4-5$ \\
\hline & \multicolumn{4}{|c|}{ Improper Fractions } \\
\hline $3 / 8$ & $5 / 4$ & $20 / 9$ & $19 / 6$ & $35 / 8$ \\
\hline $4 / 7$ & $13 / 8$ & $5 / 2$ & $17 / 5$ & $14 / 3$ \\
\hline $5 / 6$ & $17 / 9$ & $14 / 5$ & $15 / 4$ & $34 / 7$ \\
\hline $1 / 19$ & $19 / 16$ & $35 / 16$ & $40 / 13$ & $61 / 14$ \\
\hline $5 / 13$ & $17 / 12$ & $37 / 14$ & $38 / 11$ & $68 / 15$ \\
\hline \multirow[t]{8}{*}{$8 / 11$} & $28 / 15$ & $48 / 17$ & $67 / 18$ & $59 / 12$ \\
\hline & \multicolumn{4}{|c|}{ Mixed fractions } \\
\hline & $11 / 4$ & $22 / 9$ & $31 / 6$ & $43 / 8$ \\
\hline & $15 / 8$ & $21 / 2$ & $32 / 5$ & $42 / 3$ \\
\hline & $18 / 9$ & $24 / 5$ & $33 / 4$ & $46 / 7$ \\
\hline & $13 / 16$ & $23 / 16$ & $31 / 13$ & $45 / 14$ \\
\hline & $15 / 12$ & $29 / 14$ & $35 / 11$ & $48 / 15$ \\
\hline & $113 / 15$ & $214 / 17$ & $313 / 18$ & $411 / 12$ \\
\hline
\end{tabular}

Note. Stimuli prompted for strategy: 9/5, 1 9/16, 32/15, 2 7/8, 41/11, 3 5/7, 92/19, 4 4/9.

\section{Magnitude Comparison}

\section{Whole Number Magnitude Comparison}

In this iPad task, participants see a fixation point for $500 \mathrm{~ms}$ followed by two single-digit numbers (1-9) shown side by side. Participants must quickly tap the larger number. They have three seconds to make the decision before the stimulus disappears. There are three practice trials with feedback before the test trials begin. There are 16 test trials, and the ratio and distance between the digits is manipulated (Bugden \& Ansari, 2011; see Table 5 for the full set of stimuli). The placement of the larger digit (right or left) was counterbalanced. Scores were 
calculated by taking the number of correct responses divided by the total time. Reliability based on response time between trials was high (Cronbach's alpha $=.874)$.

\section{Table 5}

Symbolic Number Comparison Stimulus Set

\begin{tabular}{ccc|ccc}
\hline Number pair & Ratio & Distance & Number pair & Ratio & Distance \\
\hline $1-8$ & 0.13 & 7 & $9-5$ & 0.56 & 4 \\
$7-1$ & 0.14 & 6 & $4-7$ & 0.57 & 3 \\
$5-1$ & 0.2 & 4 & $5-8$ & 0.63 & 3 \\
$2-9$ & 0.22 & 7 & $3-2$ & 0.67 & 1 \\
$2-7$ & 0.29 & 5 & $8-6$ & 0.75 & 2 \\
$9-3$ & 0.33 & 6 & $7-9$ & 0.78 & 2 \\
$3-8$ & 0.38 & 5 & $4-5$ & 0.8 & 1 \\
$8-4$ & 0.5 & 4 & $9-8$ & 0.89 & 1 \\
\hline
\end{tabular}

Note. Small distance: difference ranged from 1 to 3 (Mean distance $=2$ ); large distance: difference ranged from 4 to 7 (Mean difference $=5$ ). Practice trials: $1-2 ; 4-3 ; 6-9$

\section{Fraction Magnitude Comparison}

Only adults completed this task. This task follows the same procedure as the whole number magnitude comparison task. Two fractions are presented side by side and participants must quickly tap the numerically larger fraction. Stimuli were proper fractions with single and double-digit denominators. These stimuli were chosen based on a previous study (Fazio et al., 2016). There are eight different problem types taken from Fazio et al. (2016) and 2 of each problem type are presented, as shown in Table 6 . These problem types were chosen to encourage the use of different strategies, and thus the participants' performance should exhibit variability in speed and accuracy. For example, on equal denominator problems, participants should be able to respond quickly by comparing the numerators. In contrast, on halves reference problems, 
participants may use a strategy that requires the processing of both the numerator and denominator in both fractions to determine which fraction is more than half and which is less than half. Participants had three practice trials with feedback to ensure that they understood the task requirements, followed by 16 test trials. As per the research literature, stimuli disappeared after 5 seconds (DeWolf, Grounds, Bassok, \& Holyoak, 2014). Scoring was the total number of correct comparisons over the total time. Reliability, calculated based on response time across trials, was moderately high (Cronbach's alpha $=.719)$.

\section{Table 6}

Examples of Each Problem Type on the Fraction Magnitude Comparison Task

\begin{tabular}{lcc}
\hline Problem type & \multicolumn{2}{c}{ Fraction Pair } \\
\cline { 2 - 3 } & Larger & Smaller \\
\hline Equal denominator & $3 / 7$ & $2 / 7$ \\
Equal numerator & $13 / 17$ & $9 / 17$ \\
& $3 / 4$ & $3 / 5$ \\
Larger numerator and smaller denominator & $2 / 13$ & $2 / 17$ \\
& $3 / 7$ & $2 / 9$ \\
Halves reference & $3 / 11$ & $2 / 15$ \\
& $2 / 3$ & $3 / 7$ \\
Multiply for common denominator & $11 / 16$ & $6 / 13$ \\
& $2 / 3$ & $5 / 9$ \\
Multiply for common numerator & $3 / 7$ & $5 / 14$ \\
& $4 / 7$ & 29 \\
Large-distance estimation (more than .30 apart) & $8 / 19$ & $4 / 15$ \\
Small-distance estimation (less than .30 apart) & 49 & $1 / 8$ \\
& $9 / 19$ & $2 / 17$ \\
& $3 / 4$ & $5 / 9$ \\
\hline
\end{tabular}

\section{Mathematical Outcome Measures}

\section{Double-Digit Arithmetic}

Children were given 1.5 minutes on each of two double-digit arithmetic pages made up of questions taken from the Calculation Fluency Test (Sowinski et al, 2014). One sheet was 
addition (e.g., $45+16$ ) and the other was subtraction (e.g., $47-12$ ). For the addition sheet, the questions were selected to ensure that there was an equal number of carrying from the one's column, the ten's column and no carrying. There were 30 questions; 10 of each type. For the subtraction sheet, questions were selected to ensure that there was an equal number of borrowing from the ten's column and no borrowing. There were 30 questions; 15 of each type. Where possible, the questions follow the order of the Calculation Fluency Test.

Adults completed the same double-digit arithmetic sheets but were only given 45 seconds per page. Scoring was the total number of correct responses divided by the time in seconds for both groups (i.e., adults and children). Reliability comparing across operations was high (Cronbach's alpha $=.865)$.

\section{Algebra}

In this task, participants are presented with a series of progressively more difficult prealgebra and algebra problems to solve. The problems were taken from the KeyMath 3 - Algebra (Connolly, 2008). Instructions for the task were: "In this task, I'm going to show you pictures of a series of problems and ask you questions about the picture I will show you. I will read the question aloud to you for you to answer. If you need me to repeat the question, then let me know." Researchers read each question and showed the corresponding picture to the participant. Students responded orally and the experimenter transcribed their answer. Testing was discontinued after three consecutive errors. A sample question is shown in Figure 3. Scoring is the total number of correct answers.

For children, questions 13 to 27 were selected to capture the earlier starting point of grade $4 \mathrm{~s}$. The starting point for Grade $4 \mathrm{~s}$ is question 13 , and for grade $6 \mathrm{~s}$ the starting point would be 15 but we had them start at the same point to compare results directly. Adults completed the same 
questions children completed as well as the remaining questions of the measure. Adults also started on item 13 but were given the opportunity to respond to questions up to 30 (i.e., the end of the test). This measure is normed up to grade 12 .

\section{Figure 3}

Example of an item similar to those on the Key Math Algebra test. The participant was asked "How would you find the value of $n$ ?"

$$
\frac{n}{4}=8
$$

\section{Fraction Knowledge Assessment}

The fraction knowledge assessment is a series of questions on both procedural and conceptual knowledge of fractions. To develop this measure, multiple sources were used. Questions were adapted from the Ontario Math Makes Sense text (Pearson, 2003) and practice booklets and the Grade 6 Education Quality and Accountability Office (EQAO) standardized test (Ontario, 1997). Jump Math (2011) support documents were also consulted. These references ensured that the questions were targeting what children were learning in their classrooms. Questions were also included from the Fraction Test Battery (Fuchs et al., 2013) and the Fraction Knowledge Assessment used by Hallett and colleagues with students in grades 6 and 8 (Hallett, Nunes, \& Bryant, 2010; Hallett, Nunes, Bryant, \& Thorpe, 2012), excluding procedures such as fraction multiplication and division that are introduced in grades 7 and 8 in Ontario.

Children. For the procedural knowledge measure, children were presented with a sheet of fraction procedures for which no knowledge of fraction arithmetic was required. These procedures involved making fraction comparisons, converting fractions to decimals, finding equivalent fractions, and converting between fraction formats (i.e., mixed numbers and improper 
fractions). Researchers demonstrated a practice problem after which the child attempted to answer as many questions as possible. This task was quite challenging and therefore children were allowed to skip questions and discontinue the task when they were struggling or simply wanted to stop. For examples of procedural questions, see Figure 4.

\section{Figure 4}

Examples of Procedural Questions.

Write fractions as decimals. Write decimals as fractions. Circle the larger fraction in the pair.
a) $\frac{3}{10}=$
e) $0.8=\frac{a}{\ldots !}$
a) $\frac{3}{7}$
$\frac{5}{7}$

For the conceptual knowledge questions, the researcher read five-word problems which were also shown on the iPad. The child answered orally, and the experimenter transcribed the response. These questions aim to assess knowledge of fraction facts and sense of fraction magnitude. For examples of conceptual questions, see Figure 5.

\section{Figure 5}

Examples of Conceptual Questions.

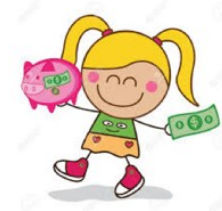

Mary and John both have some pocket money. Mary spends $1 / 4$ of hers. John spends $1 / 2$ of his.

a) Is it possible that Mary spent more than John?

b) Explain
Estimate the sum of $\frac{12}{13}+\frac{7}{8}$. Which number is closest to the answer?
a) 1
b) 2
c) 19
d) 21

Adults. Adults completed all the same questions as children as well as an additional 9 questions from the Fraction Knowledge Assessment. Both conceptual and procedural questions were completed on an iPad via Qualtrics. Participants were given pencil and paper if they needed 
it to complete any rough work to solve the problems. For the full adult Fraction Knowledge Assessment, see Appendix C. Adults received two scores for this measure: a score for questions children were given and a score for the total measure. Scoring was the total number of correct responses given.

\section{Fraction Number Line Strategy Coding}

Before testing began, research was done on the kinds of strategies children and adults use to complete fraction tasks. A coding scheme was developed based on strategies people used for placing fractions on a number line (Hannula, 2003) and making fraction magnitude comparisons (Dewolf \& Vosniadou, 2015; Fazio et al., 2016; Reys, Kim, \& Bay, 1999).

During testing, experimenters transcribed and coded the students' responses. Next, responses were transferred from the hard copy testing sheets to Excel. These responses were coded a second time by a different researcher; both codes were entered. In this second wave, incorrect strategy implementations were identified as well as a new strategy that used alternate benchmarks. For the complete strategy list, see Table 7.

The strategy list was finalized and reviewed by two researchers (Chang Xu and Sabrina Di Lonardo) who are very familiar with number line strategies. After testing with the $0-5$ fraction number line, coding was adjusted to fit the new strategy reports that did not neatly fit into the strategy coding scheme that was used for the $0-1$ fraction number line. Strategy reports were coded once during testing, and a second time by a different research assistant after testing was complete. During the second wave of coding, new strategies were more clearly defined. Table 8 shows the finalized list of strategy codes for the 0-5 fraction number line. Using this revised list, coding was completed a third time as a validity check by a different researcher. 
Reliability was calculated to ensure coders are consistent. Interrater reliability was high for both the 0-1 fraction number line and the 0-5 fraction number line respectively (Fleiss' kappa $=.905$, Fleiss' kappa $=.862$ ). Any differences were addressed based on consensus. Reconciling coding strategy discrepancies happened in steps. First, if a newly added strategy fit better with the child's response description then this strategy was selected. Second, if there were still discrepancies, the majority decision was selected. Finally, an additional coder reviewed the 12 remaining differences. These strategies will be discussed in detail in the results section.

\section{Table 7}

Final Strategy Codes for the 0-1 Fraction Number Line

\section{\# Strategy}

\section{$1 \quad$ Midpoint Reference}

2 Endpoint Reference

3 Segmenting the number line based on the denominator

4 Transforming fraction into decimal, percentage

5 Incorrect Strategies (Reference, Segmenting, Transformation etc.) 


\section{Table 8}

Revised Final Strategy Codes for the 0-5 Fraction Number Line

\# Strategy

1 Midpoint Reference

2 Endpoint Reference

3 Segmenting the number line based on the denominator

4 Transforming fraction into decimal, percentage, fraction

5 Estimation

6 Incorrect Strategies (Reference, Segmenting, Transformation, etc.)

\section{Coding Strategy Flexibility}

In order to further understand the relationships between the fraction number line tasks and other areas of mathematics, I looked at the number of strategies used in the fraction number line tasks for both children and adults. I assigned a strategy flexibility score that ranges from 0-4 for the $0-1$ fraction number line and $0-8$ for the $0-5$ fraction number line. This score is the number of different strategies each participant used. This is an individual score that was assessed for children and adults separately. Children received a score from 0-4 on the 0-1 fraction number line task. Adults received two scores: one score for the $0-1$ fraction number line and one score for the 0-5 fraction number line. For example, if Participant A used the same strategy consistently, they receive a score of 1 , whereas if participant B used 4 different strategies, they receive a score of 4 . The higher the number would mean that more strategies were used to place the fractions. 
If strategies were incorrectly used, they did not count towards the strategy flexibility score because incorrect use of strategy does not demonstrate additional knowledge. To assess incorrect strategy use, the participant would have to indicate incorrect information in their strategy report. For example, if for fraction $3 / 7$ a participant indicated that they looked at the midpoint 5 to make their estimate, this was deemed an incorrect strategy as the midpoint is $1 / 2$ or 3.5 on the $0-1$ fraction number line. Thus, if participant A used an incorrect midpoint reference to position one fraction but corrected themselves on the next trial and used the correct midpoint reference for the other fractions, this participant received a score of 1 for strategy flexibility. Moreover, if participant A correctly identified the midpoint on one trial, then incorrectly used a segmenting strategy for the following trial this participant was given a score of 1 . Strategy flexibility captures the range of participants' knowledge of different fraction concepts, any obvious inaccuracy did not count towards the score. This measure of strategy flexibility is used in the following analyses. 


\section{Results}

\section{Analysis Overview}

First, I report the effects of grade in relation to performance on number line tasks, strategy flexibility, and other measures. Tukey's HSD test was used for post-hoc comparisons.

Second, I discuss the simple correlations among measures by grade. Third, I provide a detailed analysis of strategy reports on the $0-1$ fraction number line, highlighting the similarities and differences across groups and stimuli. Finally, I analyze strategy reports on the 0-5 fraction number line and compare the effects of different types of stimuli on adults' performance.

\section{Effects of Grade}

Tables 9 and 10 provide the means, standard deviations, minimums/maximums, and skew for each of the tasks by grade.

\section{Number Line Tasks}

As shown in Table 9, adults were more accurate than grade 4 or 6 children on the $0-1000$ and the 0-1 number lines. Furthermore, PAE increased for each group with increasing number line complexity (0-1000, 0-1, 0-5). For each group, number line accuracy was positively skewed, as expected, because there is fixed minimum (i.e., 0) for this task. However, for grade 4 students, the skew was significant for the $0-1000$ number line $(z$-score $=3.81)$ but not for the $0-1$ fraction number line $(z$-score $=1.96)$. For grade $6 \mathrm{~s}$, the skew was significant for the $0-1000$ number line $(z$-score $=5.35)$ and for the $0-1$ fraction number line $(z$-score $=4.15)$. For adults the skew was significant for the $0-1000$ number line $(z$-score $=2.25)$, for the $0-1$ fraction number line $(z$-score $=6.04)$, and for the 0-5 fraction number line $(z$-score $=3.98)$. 
Table 9

Descriptive Data for Number Line Tasks (Percentage Absolute Error)

\begin{tabular}{|c|c|c|c|c|c|c|}
\hline Task & Grade & Mean & $S D$ & Min. & Max. & Skew \\
\hline \multicolumn{7}{|c|}{ Integer Number Line $0-1000$} \\
\hline \multirow[t]{3}{*}{ Error Scores (PAE) } & 4 & 11.96 & 6.51 & 3.25 & 30.41 & 1.14 \\
\hline & 6 & 7.36 & 2.98 & 3.99 & 17.45 & 1.58 \\
\hline & Adult & 3.72 & 1.27 & 1.40 & 7.97 & 1.10 \\
\hline \multicolumn{7}{|c|}{ Fraction Number Line $0-1$} \\
\hline \multirow[t]{3}{*}{ Error Scores (PAE) } & 4 & 16.79 & 11.33 & 2.95 & 43.95 & .59 \\
\hline & 6 & 10.13 & 7.06 & 2.23 & 30.04 & 1.23 \\
\hline & Adult & 6.56 & 5.11 & 2.62 & 25.44 & 2.77 \\
\hline \multicolumn{7}{|l|}{ Strategy Flexibility } \\
\hline & 4 & 1.92 & .90 & 0 & 4 & -.12 \\
\hline & 6 & 2.02 & .87 & 0 & 4 & .26 \\
\hline & Adult & 2.21 & 1.13 & 0 & 4 & .20 \\
\hline \multicolumn{7}{|c|}{ Fraction Number Line $0-5$} \\
\hline Error Scores (PAE) & Adult & 11.07 & 7.61 & 3.65 & 35.02 & 1.82 \\
\hline Strategy Flexibility & Adult & 2.39 & 1.26 & 0 & 6 & .74 \\
\hline
\end{tabular}

Note. $N \mathrm{~s}=66,64$, and 30 , for grade 4,6 , and adults, respectively.

Percentage absolute error was analyzed in a 3 (grade: 4, 6, adult) by 2 (number line type: 0-1000, 0-1) mixed ANOVA. There was a main effect of number line type, $F(1,157)=24.20, p$ $<.001, \eta_{p}{ }^{2}=.13$. Participants were less accurate on the $0-1$ fraction number line $(11.23 \%)$ than on the $0-1000$ number line $(7.69 \%)$. Moreover, there was a significant main effect of grade, $F(2$, $157)=33.32, p<.001, \eta_{p}{ }^{2}=.30$. Post-hoc Tukey’s HSD test revealed that adults performed better $(5.14 \%)$ than both groups of children and grade 6 students $(8.75 \%)$ also performed better than Grade 4 students (14.38\%). However, there was no significant interaction between number line type and grade, $F(2,157)=1.18, p=.31, \eta_{p}{ }^{2}=.02$ (see Figure 7$)$. Thus, as predicted in 
Hypothesis 1 and 2, adults performed better on both number lines than both groups of children and Grade 6 students performed better on both number lines than Grade 4 students. Furthermore, the $0-1$ fraction number line was harder than the $0-1000$ number line for all three groups. This evidence supports hypothesis 6 .

\section{Figure 6}

Mean Percentage Absolute Error on Integer and Fraction Number Lines by Grade

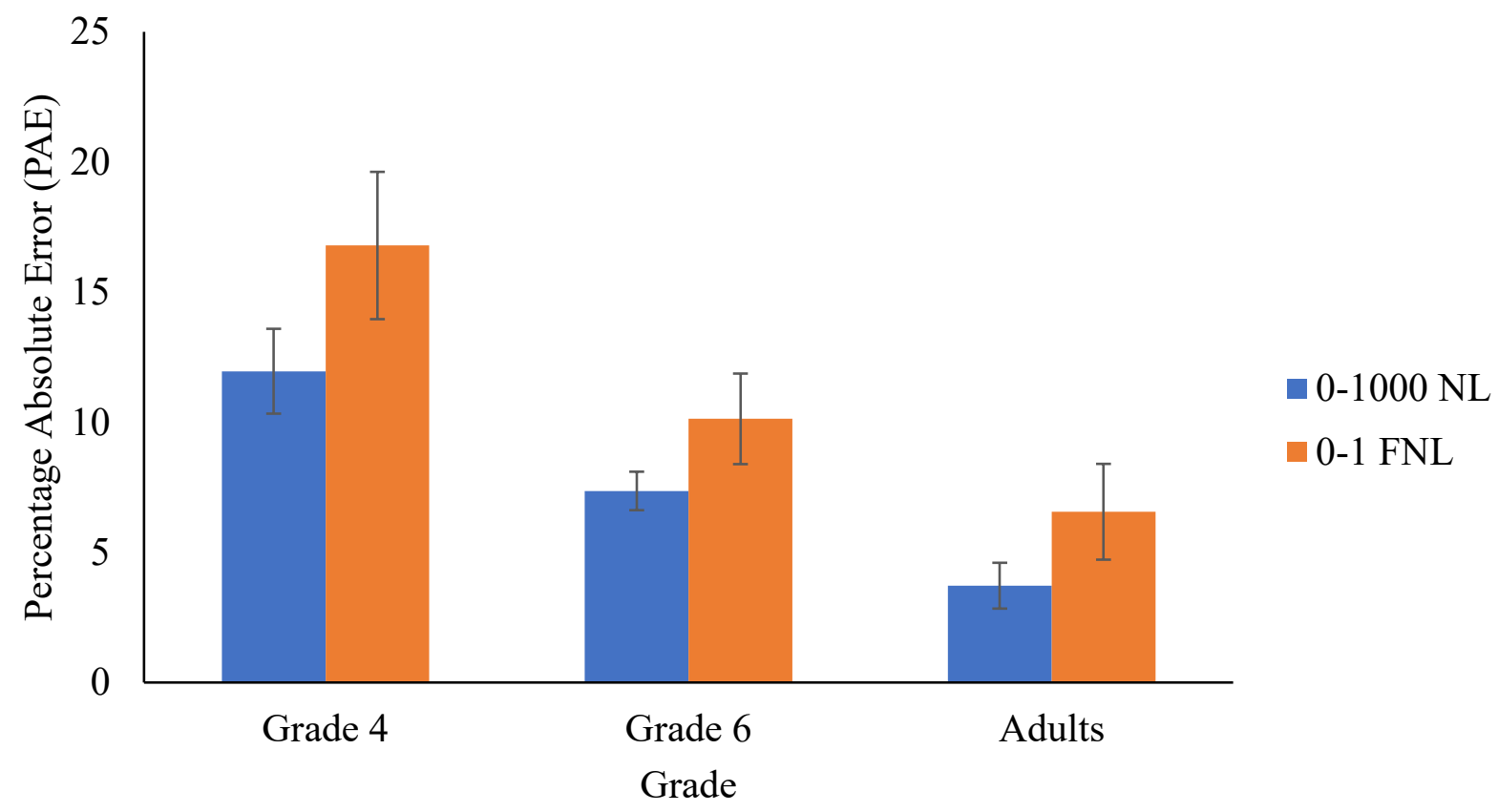

Note. Error bars are $95 \%$ confidence intervals of the mean.

\section{Grade Effects on Other Measures}

Participants' performance by grade on all of the other measures is shown in Table 10. Adults had higher scores than children on each of the measures and grade 6 students had higher scores than grade 4 students. Performance on each measure was analyzed in a 3 (grade: 4, 6 , adult) ANOVA. There were significant effects of grade for integer magnitude comparison, $F(2$, $157)=91.63, p<.001, \eta_{p}{ }^{2}=.55$, total arithmetic fluency score, $F(2,157)=64.03, p<.001, \eta_{p}{ }^{2}=$ .45 , fraction knowledge assessment, $F(2,157)=71.95, p<.001, \eta_{p}{ }^{2}=.48$, algebra, $F(2,157)=$ 
$14.91, p=<.001, \eta_{p}{ }^{2}=.16$, and digit span backwards, $F(2,157)=28.85, p=<.001, \eta_{p}{ }^{2}=.27$. Post-Hoc Tukey's HSD revealed there was no significant difference in performance between grade $4 \mathrm{~s}$ and grade $6 \mathrm{~s}$ on digit span backwards or algebra. However, as expected in Hypothesis 1 and 2, there was a significant effect of grade for all other measures in the predicted direction. 


\section{Table 10}

Descriptive Data for Cognitive, Numerical, and Fraction Tasks

\begin{tabular}{lcccccc}
\hline Task & Grade & $M$ & SD & Min & Max & Skew \\
\hline Integer Comparison $^{1}$ & 4 & .98 & .15 & .58 & 1.30 & -.14 \\
& 6 & 1.14 & .18 & .67 & 1.51 & -.04 \\
Fraction Comparison $^{1}$ & Adult & 1.54 & .21 & 1.10 & 1.96 & .10 \\
Algebra $^{2 *}$ & Adult & 3.63 & 1.49 & 1.60 & 5.00 & 1.04 \\
& 4 & 4.09 & 2.08 & 0 & 9 & .23 \\
& 6 & 5.95 & 2.84 & 0 & 12 & .10 \\
Fraction Knowledge Assessment $^{2 *}$ & Adult & 16.53 & 7.84 & 3 & 27 & -.44 \\
& 4 & 8.45 & 4.71 & 2 & 23 & 1.34 \\
& 6 & 16.94 & 8.40 & 4 & 32 & .11 \\
Addition Fluency $^{1}$ & Adult & 36.97 & 8.22 & 17 & 48 & -.97 \\
& 4 & .10 & .05 & .01 & .24 & .49 \\
& 6 & .15 & .06 & .02 & .29 & .14 \\
Subtraction Fluency $^{1}$ & Adult & .29 & .12 & .11 & .62 & 1.19 \\
& 4 & .06 & .05 & 0 & .28 & 2.43 \\
& 6 & .08 & .04 & 0 & .19 & .44 \\
Digit Backwards $^{2}$ & Adult & .17 & .10 & .04 & .40 & .86 \\
& 4 & 5.98 & 1.47 & 3 & 11 & .44 \\
& 6 & 6.92 & 1.66 & 3 & 11 & .42 \\
& Adult & 9.60 & 3.24 & 6 & 18 & .88 \\
\hline
\end{tabular}

Note. The metrics for each task were as followed ${ }^{1}$ number of correct responses / time (in seconds), ${ }^{2}$ number of correct responses.

* = tests have added questions for adults 


\section{Correlations}

Adults

Correlational data for all of the measures are shown in Table 11 for the adults (above the diagonal). First, consider the three number line tasks. Significant correlations were found between the 0-1000 and 0-5 number lines, whereas performance on the $0-1$ number line was not correlated with either of the other number lines. After inspection of the scatterplots, it appears that there are two extreme scores (i.e., $>3$ SDs above the mean) on the $0-1$ fraction number line. Upon removing these extreme scores, there was a large positive correlation between the 0-1 fraction number line and the 0-5 fraction number line. The revised correlations are also shown in Table 11 (below the diagonal). However, the correlation between the $0-1000$ number line and the 0-1 fraction number line was still not significant. 


\section{Table 11}

Correlational Data for Adults $(N=30)$ (above the diagonal), correlations omitting outliers $(N=28$; below the diagonal)

\begin{tabular}{|c|c|c|c|c|c|c|c|c|c|c|c|}
\hline & 1 & 2 & 3 & 4 & 5 & 6 & 7 & 8 & 9 & 10 & 11 \\
\hline 1 Integer NL 0-1000 & - & .144 & .258 & -.239 & -.114 & -.162 & -.103 & -.133 & -.355 & -.239 & -.206 \\
\hline 2 Fraction NL 0-1 & .198 & - & .240 & $-.414^{*}$ & $.475^{*}$ & -.148 & -.322 & -.080 & -.227 & $-.512 * *$ & .129 \\
\hline 3 Fraction NL 0-5 & $.408^{*}$ & $.718^{* *}$ & - & $-.501 * *$ & -.018 & -.326 & $-.735 * *$ & $-.652 * *$ & -.299 & -.226 & -.249 \\
\hline 4 Integer MagComp & -.204 & -.317 & $-.501 * *$ & - & -.132 & .364 & $.490 * *$ & $.407^{*}$ & .187 & $.478 * *$ & -.123 \\
\hline 5 Fraction MagComp & -.242 & -.097 & -.018 & -.132 & - & .273 & -.049 & .042 & .167 & -.118 & .205 \\
\hline 6 Arithmetic & -.132 & -.319 & -.326 & .364 & .273 & - & $.402 *$ & $.626 * *$ & $.440 *$ & $.416^{*}$ & .106 \\
\hline 7 Fraction KA & -.028 & $-.580^{* *}$ & $-.735 * *$ & $.490 * *$ & -.049 & $.402 *$ & - & $.650 * *$ & .322 & $.405 *$ & .114 \\
\hline 8 Algebra & .013 & $-.426^{*}$ & $-.652^{* *}$ & $.407^{*}$ & .042 & $.626^{* *}$ & $.650 * *$ & - & .189 & .062 & .226 \\
\hline 9 Digit Back & -.298 & -.200 & -.299 & .187 & .167 & $.440^{*}$ & .322 & .189 & - & .333 & .165 \\
\hline 10 Str.Flex 0-1 NL & -.141 & -.369 & -.226 & $.478^{*}$ & -.118 & $.416^{*}$ & .368 & .062 & .333 & - & -.113 \\
\hline 11 Str. Flex 0-5 NL & .042 & -.196 & -.249 & -.123 & .205 & .106 & .114 & .226 & .165 & -.113 & - \\
\hline
\end{tabular}

$* p<.05, * * p<.01$ 
The relations between number line performance and other measures are also important. As shown in Table 11, the 0-1 fraction number line was correlated with both the integer magnitude comparison performance and the fraction magnitude comparison. However, when outliers were removed, these correlations were no longer significant and instead, the $0-1$ fraction number line was correlated with performance on the algebra and fraction knowledge assessments. The 0-5 fraction number line was also correlated with performance on integer magnitude comparison as well as the algebra and fraction knowledge assessments. Interestingly, performance on the $0-1000$ number line was not related to other measures. Moreover, performance on the fraction magnitude comparison measure was not significantly correlated with any other measure. In contrast, the integer magnitude comparison was positively correlated with both the algebra and fraction knowledge assessments. This difference could be attributed to the reaction times of the integer magnitude comparison task compared to the more active processing longer response times of the fraction magnitude comparison task. However, some of the effect sizes were moderate and thus, in interpreting these correlations, we have to keep in mind the small sample size.

\section{Grade 4 and Grade 6}

Correlations among all the measures for Grade 4 (above the diagonal) and Grade 6 students (below the diagonal) are shown in Table 12. Error scores on both the 0-1000 number line and the 0-1 fraction number line were negatively correlated with all of the math outcome measures (i.e., arithmetic, fraction knowledge assessment, and algebra) for both grades, however, in grade 6 , the correlation between the integer number line and arithmetic was not significant. The correlations between the 0-1000 number line and integer magnitude comparison were significant for both groups. Interestingly, for grade 4 students, performance on the integer 
number line was highly correlated with digit backwards span, whereas for grade 6 students this correlation was not significant. Otherwise, the pattern of correlations between grades is very similar for both number lines.

\section{Table 12}

Correlations for Children in Grade 4 (above the diagonal, $N=64$ ) and Grade 6 (below the diagonal $N=66$ )

\begin{tabular}{lcccccccc}
\hline & 1 & 2 & 3 & 4 & 5 & 6 & 7 & 8 \\
\hline 1 Integer NL 0-1000 & - & $.568^{* *}$ & -.256 & $-.439^{* *}$ & $-.371^{* *}$ & $-.548^{* *}$ & $-.277^{* *}$ & .088 \\
2 Fraction NL 0-1 & $.587^{* *}$ & - & -.155 & $-.471^{* *}$ & $-.549^{* *}$ & $-.523^{* *}$ & $-.399^{* *}$ & .037 \\
3 Integer MagComp & $-.354^{* *}$ & $-.282^{* *}$ & - & .130 & .075 & .096 & .111 & .005 \\
4 Arithmetic & -.194 & $-.420^{* *}$ & .230 & - & $.492^{* *}$ & $.531^{* *}$ & $.495^{* *}$ & .041 \\
5 FKA & $-.383^{* *}$ & $-.636^{* *}$ & .174 & $.582^{* *}$ & - & $.578^{* *}$ & $.319^{*}$ & $-.255^{*}$ \\
6 Algebra & $-.422^{* *}$ & $-.560^{* *}$ & $.425^{* *}$ & $.418^{* *}$ & $.565^{* *}$ & - & $.374^{* *}$ & -.192 \\
7 Digit Back & -.078 & $-.308^{*}$ & $.309^{*}$ & $.401^{* *}$ & $.397^{* *}$ & $.287^{*}$ & - & .071 \\
8 Strategy Flexibility & .231 & $.249^{*}$ & -.012 & -.029 & -.116 & -.068 & -.021 & - \\
\hline
\end{tabular}

$* p<.05, * * p<.01$

Note. FKA is Fraction Knowledge Assessment. 


\section{Strategy Flexibility}

For adults, strategy flexibility on the 0-1 Fraction Number line, as measured by the number of different strategies used on strategy report trials, was positively correlated with performance on the $0-1$ fraction number line (with outliers). When outliers are removed this correlation is no longer significant. Furthermore, as predicted in Hypothesis 3, strategy flexibility on the 0-1 Fraction Number Line was positively correlated with performance on arithmetic, fraction knowledge assessment and integer magnitude comparison. However, contrary to Hypothesis 3 , strategy flexibility did not correlate with performance on the Algebra assessment. Strategy flexibility on the $0-5$ fraction number line did not correlate significantly with any measure. This unexpected finding could be the result of participants rounding to the nearest whole number more than actively processing the fractions which I will discuss in the strategy reports section for the 0-5 fraction number line.

For Grade 4 children, strategy flexibility was correlated with performance on the Fraction Knowledge Assessment. Children who used more strategies had lower scores on the FKA, suggesting that strategy variability reflected a lack of fraction understanding at this grade level. For Grade 6 children, strategy flexibility was positively correlated with performance on the $0-1$ fraction number line. However, there were no correlations with mathematical outcome measures which was contrary to Hypothesis 3 . Although children in grade 6 demonstrate better fraction knowledge from children in grade 4 , they had limited knowledge of strategies available to them or they may not have enough practice with different strategies required for automaticity. For a strategy to be selected over an easier strategy, the problem solver needs to be as confident in their ability to quickly and accurately implement the strategy as they would be for the easier strategy. With practice and experience, adults can make the transition to more difficult strategies 
as they begin to rely on these strategies for efficiency, the implementation becomes automatic. The need for automaticity in strategy selection is more salient in a timed task.

\section{0-1 Fraction Number Line}

\section{Accuracy}

Before analyzing the strategies, I compared performance on the four items for which participants provided strategy reports. Percent absolute error was analyzed in a 3(grade: 4, 6, adult) by 4(fraction: $1 / 4,3 / 7,4 / 5,7 / 9$ ) mixed ANOVA. Performance varied across the four fractions, $F(3,468)=6.16, p<.001, \eta_{p}{ }^{2}=.04$, as shown in Figure 7. Post Hoc comparisons using Tukey's HSD showed multiple significant differences between fractions. Fractions 1/4 and 7/9 were placed significantly more accurately than fraction $4 / 5$ and $3 / 7$. However, there was no difference between $1 / 4$ and $7 / 9$.

\section{Figure 7}

Accuracy Collapsed across grade for each fraction on the 0-1 fraction number line.

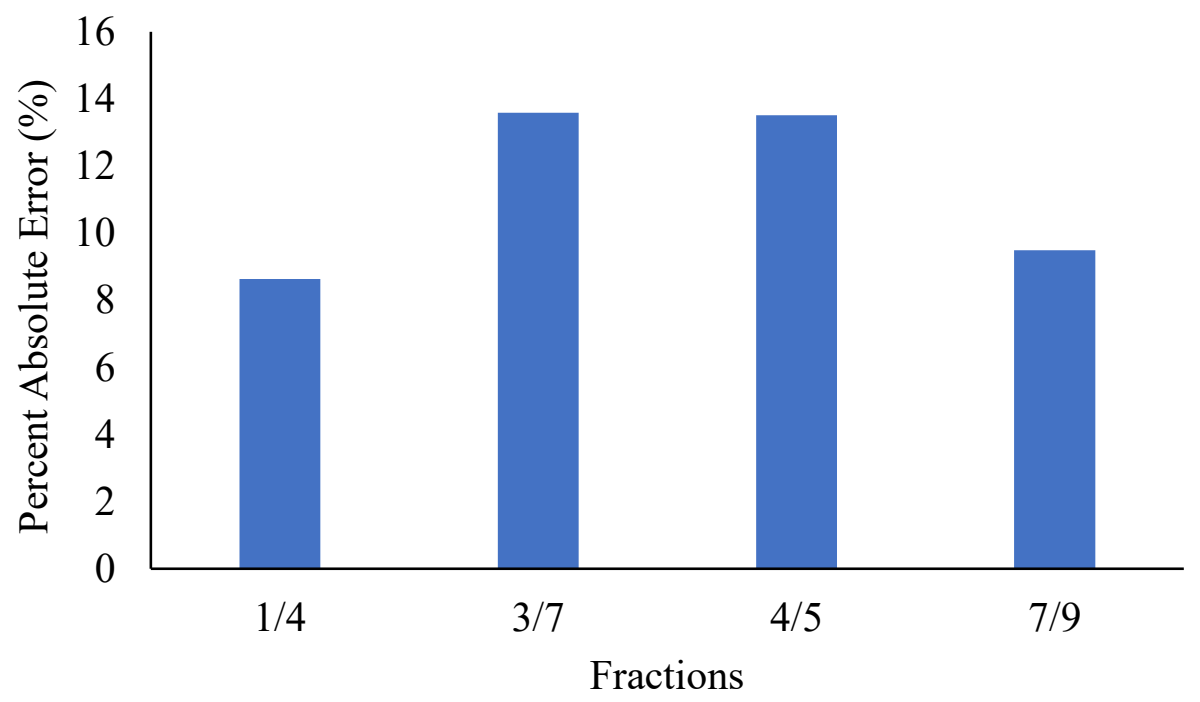

There was a main effect of grade, $F(1,156)=274.2, p<.001, \eta_{p}{ }^{2}=.64$. Post Hoc Tukey's test revealed that adults $(7.44 \%)$ and grade $6 \mathrm{~s}(9.94 \%)$ performed better than grade $4 \mathrm{~s}(16.56 \%)$. 
However, there was no significant difference in performance between adults and grade $6 \mathrm{~s}$. There was no significant interaction of fraction by grade, $F(3,468)=1.57, p=.154, \eta_{p}{ }^{2}=.02$. Grade 4 children always had the least accurate performance. However, this interaction suggests that grade 6 children are somewhat worse than adults on $3 / 7$ and $4 / 5$, the fractions closer to the middle of the line. Adults showed quite similar performance across the four fractions whereas children tended to be less accurate on the fractions that were in the middle.

\section{Figure 8}

Accuracy by grade for placing the target numbers on the 0-1 fraction number line.

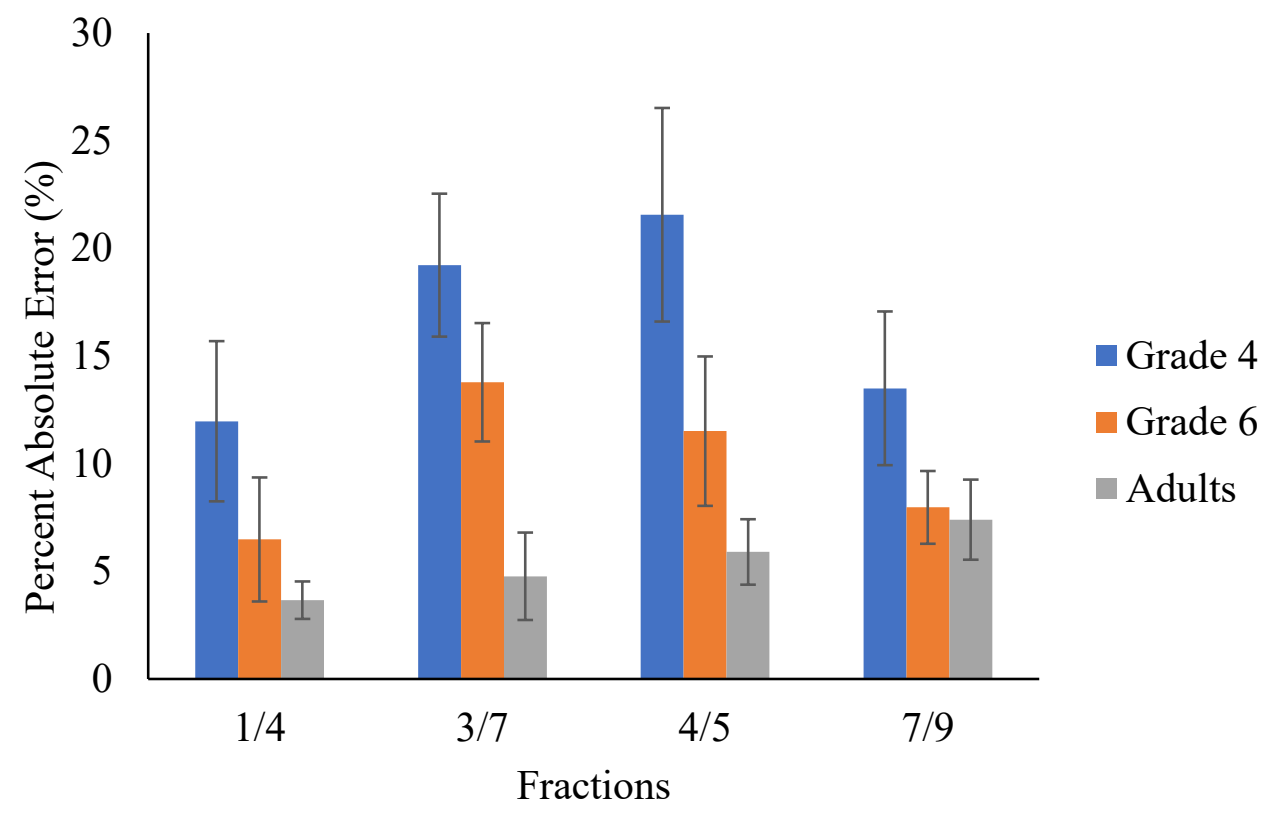

Note. Error bars are $95 \%$ confidence intervals

\section{Strategy Reports}

Comparing strategy reports for the 0-1 fraction number line showed interesting differences between children in grades 4 and 6, and adults.

One fourth. For fraction $1 / 4$, strategy use varied with grade, $X^{2}(8, N=160)=24.61, p=$ 
.002. As shown in Figure 9, most of the adults used a segmenting strategy to make their estimates. In contrast, although the most common strategy for both grades 4 and 6 students was also segmenting, they also reported more incorrect strategies (especially grade 4), and more midpoint strategies (especially grade 6). Adults' segmenting strategies included statements such as "half of a half" or "split the line into 4". Interestingly, adults' incorrect strategies often involved segmenting, albeit incorrectly, as in "I counted to 4 and placed it there", or "two halves of two halves." The children's correct segmenting strategies were similar to those of adults. They also occasionally used midpoint strategies, such as "less than half" and "close to half". However, children's incorrect strategies were more varied than those of adults including the use of whole number strategies to place fractions on the number line $(20 \%)$, use of an incorrect midpoint $(5 \%)$, use of an incorrect counting strategy (5\%) (i.e., $1 / 2,1 / 3,1 / 4,1 / 5$, using whole number rules based on the denominator) or responding “I don't know". In summary, adults made fewer strategy errors than children, but the dominant correct strategy was segmenting for all three groups. 


\section{Figure 9}

Percentage of Students in Each Grade: Strategies for Placing 1/4

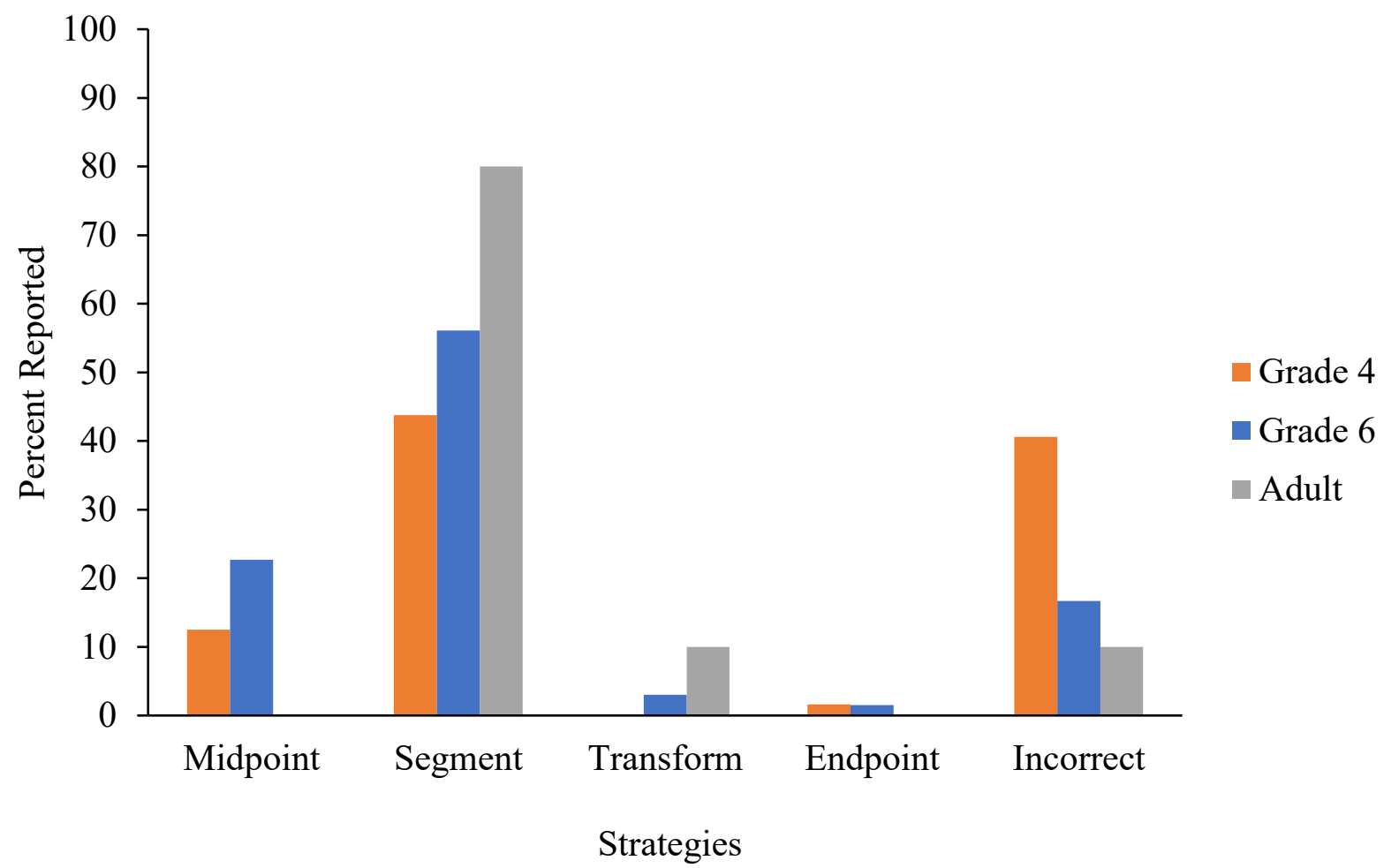

Three sevenths. For fraction $3 / 7$, strategy use varied with grade, $X^{2}(8, N=160)=$ 44.94, $p<.001$. As shown in Figure 10, most adults used a midpoint strategy to make their estimates of 3/7. In contrast, the most common strategy for children in both grade 4 and grade 6 was the segmenting strategy. Moreover, children reported drastically more incorrect strategies than adults. Adults' use of the midpoint reference included (50\%) indicating the midpoint as $3.5 / 7$ however $10 \%$ of students used other similar fractions to estimate $3 / 7$. Other similar fractions reported as the midpoint were $3 / 6(7 \%)$ and 4/8 (3\%) suggesting even numbers may allow for a clearer division of the number line into half for these students. The use of the segmenting strategy was similar to that of the previous fraction "split the line into 7ths" (20\%). A transformation strategy was used by $13 \%$ of adults to make their estimates for $3 / 7$. The use of this strategy involved altering the numerator or denominator of fractions (7\%) (e.g., double the 
numerator and you get almost a whole so just below 50\% of the line), transforming the fraction to a decimal (3\%) and transforming the fraction to a percentage (3\%). Incorrect strategies were used in 7\% of adult reports which included: "3 fits into 7 four times so I placed it close to 4 " and " $3 / 7$ is .2 " an incorrect transformation strategy.

Interestingly, for students in grades 4 and 6 , the preferred strategy was the same as the preferred strategy for $1 / 4$ (i.e., segmenting). Furthermore, in both grades there was a $20 \%$ increase in incorrect strategies used to estimate $3 / 7$ than there were for the fraction $1 / 4$, including "I don't know" and "I guessed" among others. The most interesting incorrect strategy is the whole number strategy. This strategy involves processing only the denominator or only the numerator of fractions and placing them as if the number line ranges from $0-10 ; 13 \%$ of students made this mistake. Other errors involved adding or subtracting the numerator and denominator from one another to reach a single number which can be processed alone (6\%). Fraction $3 / 7$ was a more challenging fraction for the children to place as it is not as familiar as $1 / 4$. For the full distribution of fraction 3/7 of strategy use by grade see Figure 10. 


\section{Figure 10}

Strategy Report Comparison for Fraction 3/7 by Grade

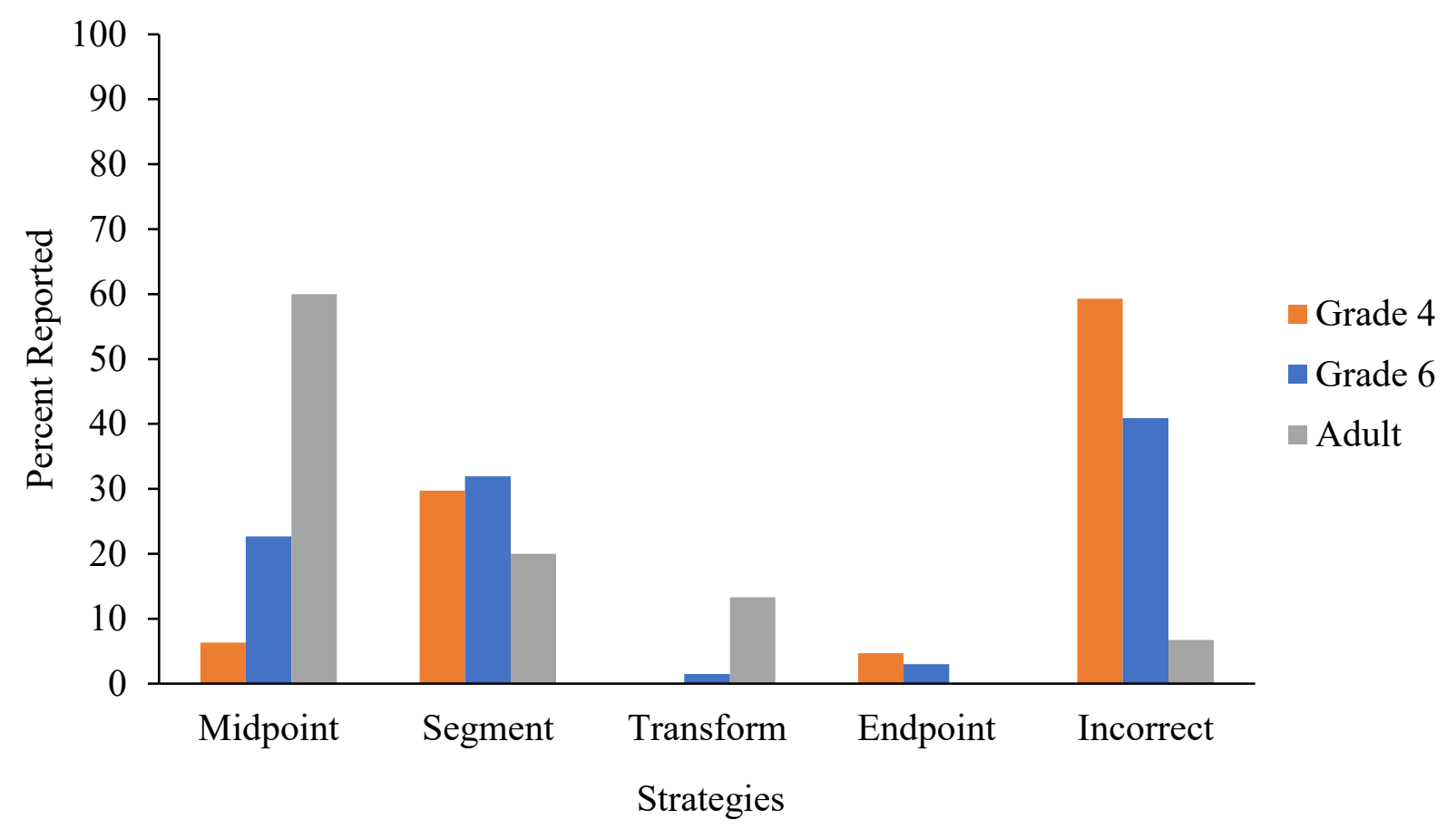

Four fifths. For fraction 4/5, strategy use varied with grade, $X^{2}(8, N=160)=$

41.172, $p<.001$. For the fraction $\mathbf{4 / 5}$, it was expected that transformation might be the most used strategy, however as shown in Figure 11, both segmenting (43\%) and transformation (37\%) were frequently used by adults. For children, the most common strategy again was the segmenting strategy for both groups. However, there was an increase in endpoint strategies for all three groups. Moreover, incorrect strategies were less frequent on this fraction than the others.

Adults used transformation more frequently than on the other fractions: $30 \%$ stated that they converted the fraction to $80 \%$ and $7 \%$ more reported dividing $4 / 5$ to get .8 . The reporting of segmenting was similar to the other fractions and similar across grades "split line into 5". Children's use of the endpoint strategy involved reporting "close to 1", "almost a whole", and "a big number, less than 1". The change of strategy from previous fractions reflects an attempt to 
gain efficiency and maintain accuracy. No children in grade 4 used transformation to make their estimates and only $3 \%$ of grade 6 students used a transformation strategy. Their strategy selection provides evidence that the skill required to transform fractions to decimals and percentages is not yet developed by these critical timepoints in a student's fraction knowledge development. Decimals are taught in grade 5 in Ontario, thus grade 6 s but not grade 4s should have some knowledge of this procedure.

\section{Figure 11}

Strategy Report Comparison for Fraction 4/5 by Grade

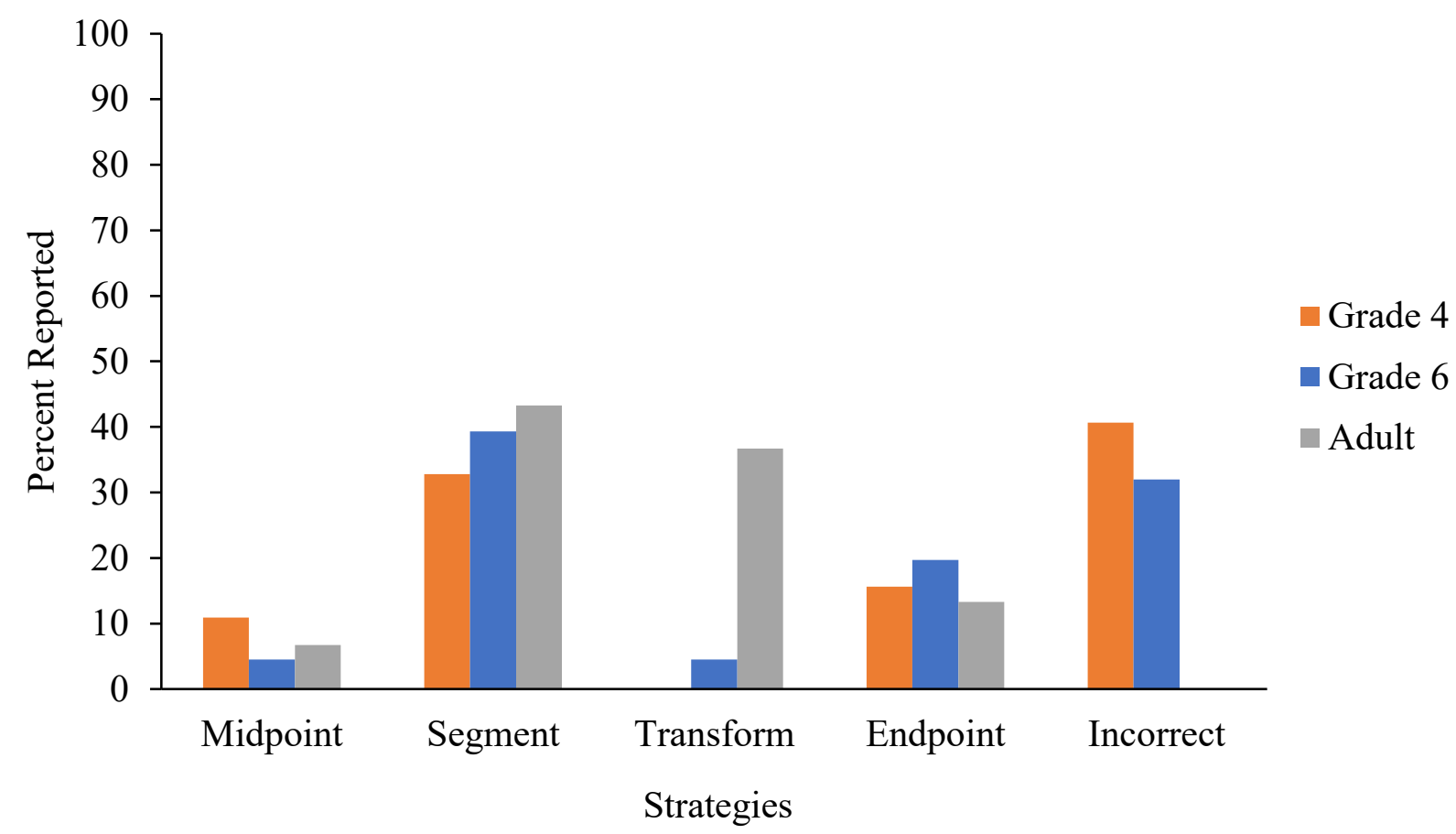

Seven ninths. For fraction $7 / 9$, strategy use varied with grade, $X^{2}(8, N=160)=$ $35.48, p<.001$. There was no obvious strategy choice to predict for fraction $\mathbf{7 / 9}$ and strategy reports reflected this (see Figure 12) -- participants used many different strategies. Adults used transformation, segmentation, and endpoint strategies. Children in grades 4 and 6 used 
segmenting and endpoint strategies. There was no obvious striking preferred strategy by any of the groups, except that grade 4 s slightly preferred segmenting again.

Transformation was the most frequently used strategy for this fraction by adults, slightly more than endpoint and segmenting. However, these transformations were not to decimal or percentage as with previous fractions but instead changing the fraction to make estimation easier. For example, $10 \%$ of participants reduced the numerator from 7 to 6 and noted that $6 / 9$ was equal to $2 / 3$ and thus put their estimate just over that point. Another $7 \%$ of participants increased the denominator from 9 to 10 and went to $7 / 10$ and placed their estimate slightly more. The endpoint strategy for adults involved reporting: "almost a whole" and "close to 1". The use of segmenting involved reporting "split into 9". Children reported segmenting and endpoint strategies similarly to the adults.

\section{Figure 12}

Strategy Report Comparison for Fraction 7/9 by Grade

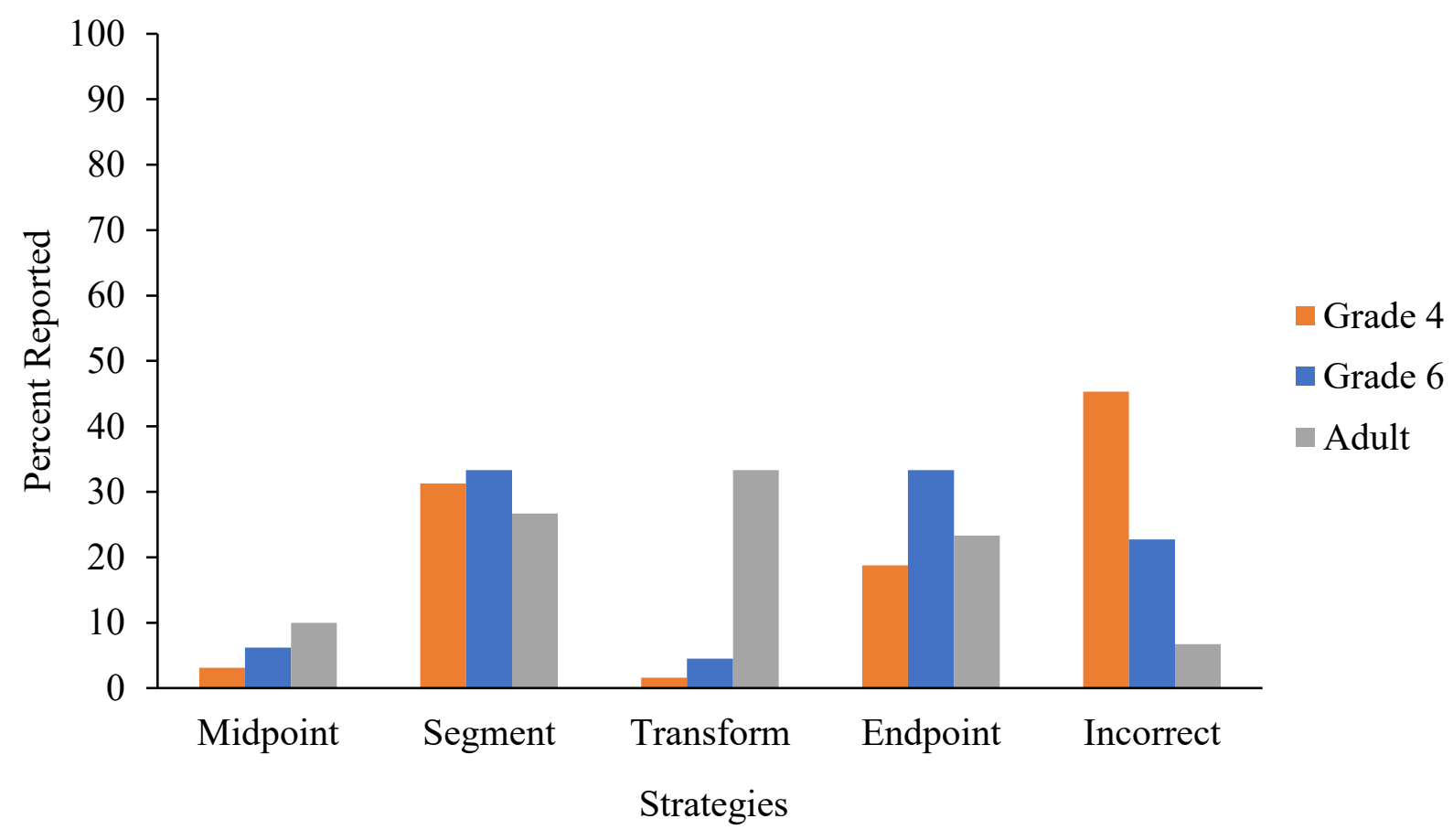


Summary. Strategy reports on each of the fractions show different patterns of use by grade, however, across fractions some patterns arise as well. For all fractions, strategy use varied by grade according to chi-squared tests. Segmenting was commonly used by all groups across fractions. Midpoint references were commonly used if fractions were before the halfway mark by all groups, and endpoint references were commonly used if fractions were after the midpoint by all groups. It appears that all participants are pulled to the nearest benchmark to the right of the target number. Adults were more likely than children to use transformation strategies, suggesting that there is a certain automaticity of transforming fractions to decimals and percentages that develops with more math education. Children start relating fractions to decimals in Grade 5 under the Ontario Math Curriculum and thus this skill is relatively new for grade 6s and not yet learnt by grade $4 \mathrm{~s}$.

Endpoint strategies were more frequently used by children than adults. Furthermore, referencing the endpoint for adults was uncommon and only reported when fractions had a high denominator. Children in both grades relied more heavily on the endpoint to make all estimates than adults. Children seemed to mention the endpoint often even while describing other strategies, possibly to remind themselves what the whole was for different denominators (i.e., the whole is 9). Grade 4s made the most errors in strategy implementation, using whole number rules to make their estimates and focusing on one part of the fraction rather than its entirety. In summary, adults demonstrated a shift in strategy preference based on the fraction. Children in both grades did not demonstrate the same flexibility to make their estimates. 


\section{Adult Number Line Analyses}

\section{Comparisons Across Number Lines}

For the following analyses, adult data was analyzed separately from children's data because only adults did the 0-5 fraction number line. Participants whose scores were deemed outliers for the 0-1 fraction number line were omitted from this analysis. To compare performance across the three different number lines, percentage absolute error was analyzed in a 3 (number line type: integer 0-1000, fraction 0-1, fraction 0-5 number line) repeated measures ANOVA. There was a main effect of number line type, $F(2,52)=30.21, p<.001$. As hypothesized, performance was better on the $0-1000$ number line $(3.72 \%)$ than on both of the fraction number lines. Furthermore, as predicted, accuracy was better on the $0-1$ fraction number line $(6.56 \%)$ than on the $0-5$ fraction number line (11.07\%). Thus, consistent with Hypothesis 3 , accuracy decreased as the complexity of the number line increased.

\section{Analyses of the 0-5 Number Line}

Only adults completed the $0-5$ fraction number line. To make this task challenging for adults, I manipulated fraction format (improper vs. mixed) as well as the denominator (single digit vs. double digit). All 30 participants were included in this analysis. To compare across stimulus types, percentage absolute error was analyzed in a 2(fraction format: improper vs. mixed number) by 2(denominator: single digit vs. double digit) repeated measures ANOVA. Contrary to Hypothesis 7 , there was no main effect of fraction type, $F(1,29)=2.14, p=.155, \eta_{p}{ }^{2}$ $=.07$. Improper fractions $(9.92 \%)$ and mixed numbers $(12.58 \%)$ were equally challenging to estimate. Participants transformed fractions between formats to suit their estimation strategies which could explain this result. These transformations will be further discussed in the strategy report section. Moreover, there was no main effect of denominator, $F(1,29)=2.83, p=.104, \eta_{p}{ }^{2}$ 
$=.09$. Estimating single-digit denominator fractions $(11.3 \%)$ and double-digit denominator fractions $(10.9 \%)$ were equally difficult. Finally, there was no interaction, $F(1,29)=.45, p=$ $.509, \eta_{p}^{2}=.02$.

Response time was also analyzed in a 2 (fraction format: improper vs. mixed) by 2 (denominator: single-digit vs. double-digit) repeated measures ANOVA. There was a significant main effect of fraction format for response time, $F(1,29)=10.95, p<.001, \eta_{p}{ }^{2}=.42$. Improper fractions took longer to estimate than mixed numbers (4138 vs. $2953 \mathrm{~ms}$ ). Thus, participants may have transformed improper fractions to a mixed number format, accounting for the extra time, however, this transformation did not result in a less accurate estimate. No main effect was found for number of digits in the denominator, $F(1,29)=.86, p=.361, \eta_{p}{ }^{2}=.03$. However, there was a significant interaction effect found for the response time of fraction format by denominator (see Figure 13), $F(1,29)=9.651, p=.004, \eta_{p}^{2}=.26$. Thus, the number of digits in the denominator did affect response time but only when the fraction was in the improper format. Participants took longer estimating the location of improper fractions with double-digit denominators. 


\section{Figure 13}

Mean Percent Absolute Error by Fraction Format and Number of Digits in the Denominator

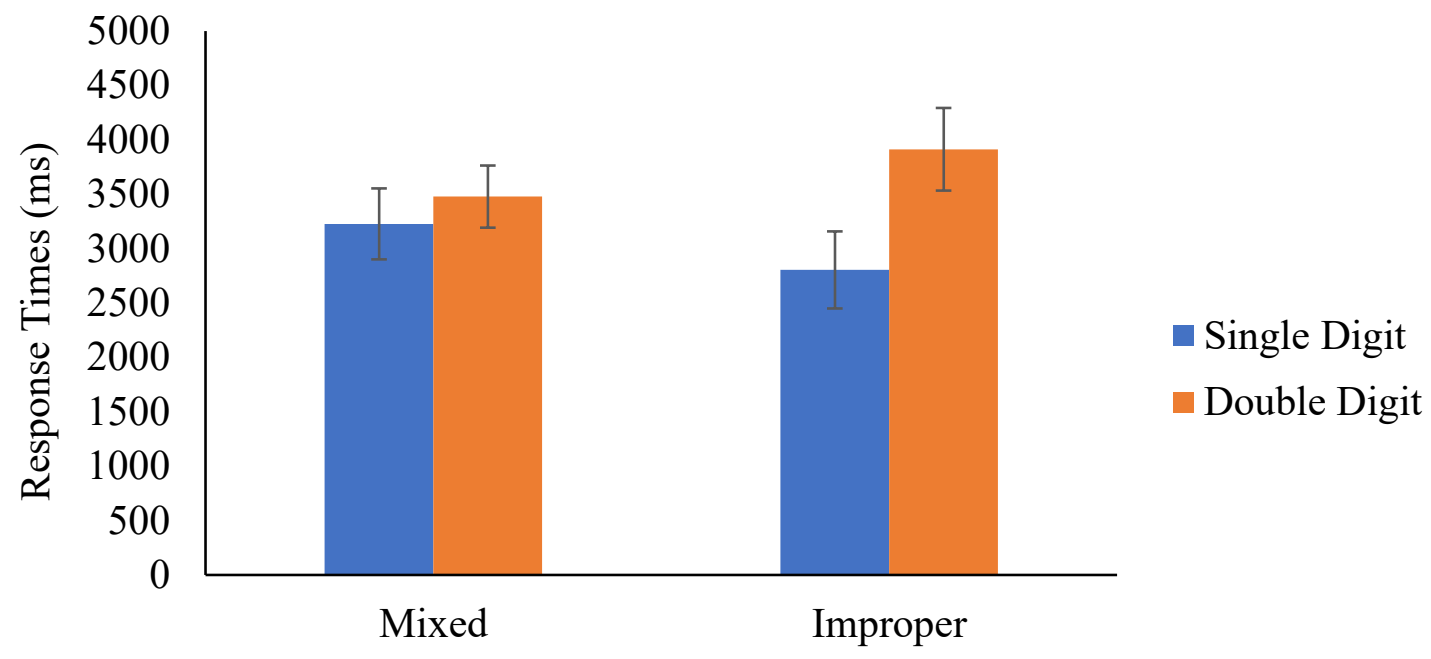

Fraction Formats

Note. Error bars are 95\% confidence intervals.

\section{Strategy Reports on the 0-5 Fraction Number Line}

The 0-5 fraction number line strategy reports were very different from those for the $0-1$ fraction number line. Eight fractions were prompted for strategy report, four mixed numbers and four improper fractions. Mean percent absolute errors for each fraction are shown in Figure 14. Percent absolute error was analyzed in an 8 (fraction: 9/5, 1 9/16, 32/15, 2 7/8, 41/11, 3 5/7, 92/19, 4 4/9) repeated measures ANOVA. There was no main effect of fraction, $F(7,196)=$ $.799, p=.589, \eta_{p}^{2}=.03$. Overall, PAE looks quite similar across the various fractions. 


\section{Figure 14}

Accuracy for Placing Target Fractions

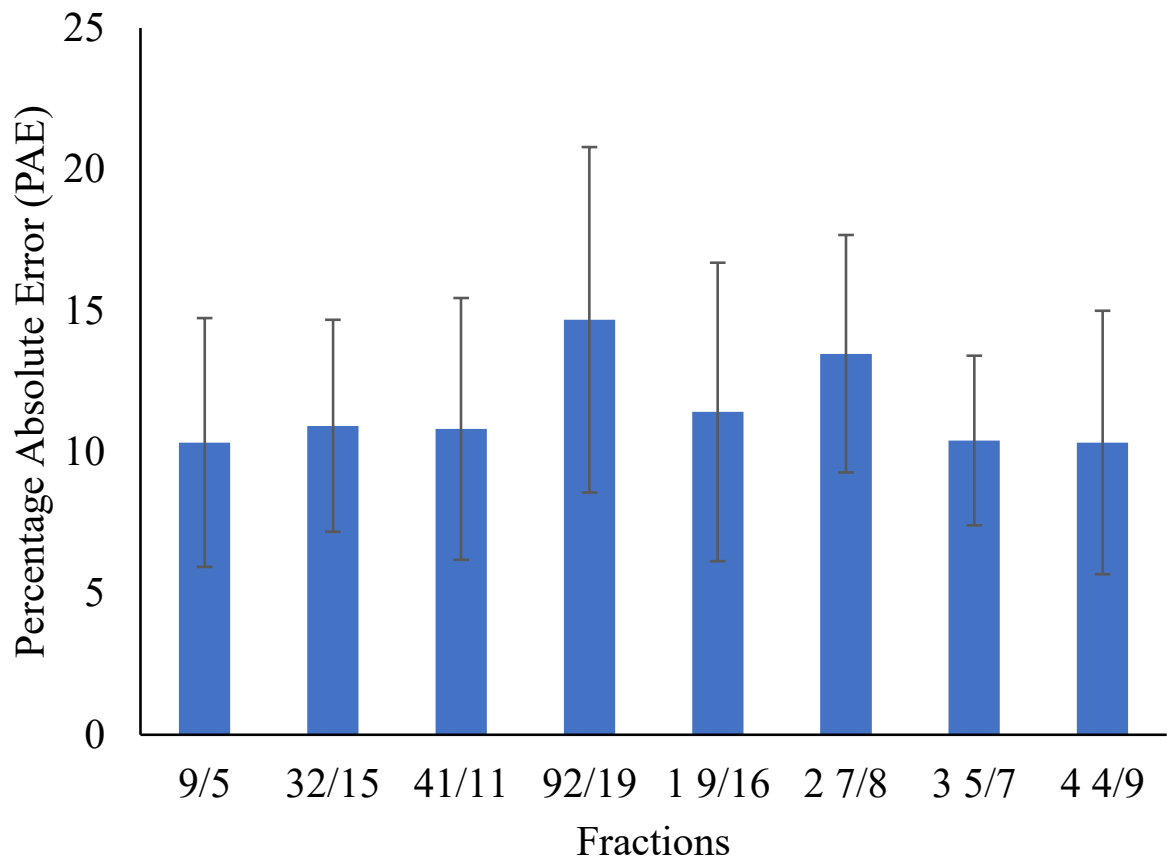

Note. Error bars are 95\% confidence intervals.

Improper Fractions. The improper fractions were 9/5, 32/15, 41/11, 92/19. The

percentage of participants who reported each strategy across these fractions is shown in Figure 15. For the fraction $\mathbf{9 / 5}$, strategy use varied $X^{2}(5, N=30)=13.2, p=.02 .37 \%$ of participants reported transforming the fraction to a mixed number to place their estimate. Of this $37 \%, 20 \%$ reported an additional strategy to place the fraction including midpoint reference $(10 \%)$ and additional transformation strategy of the remainder fraction to a percent or decimal (10\%). 17\% of participants estimated and reported "almost 2" or "higher than 1" with no other strategy following these reports even after being prompted a second time. 


\section{Figure 15}

Strategies Reported on the 0-5 Fraction Number Line for Improper Fractions

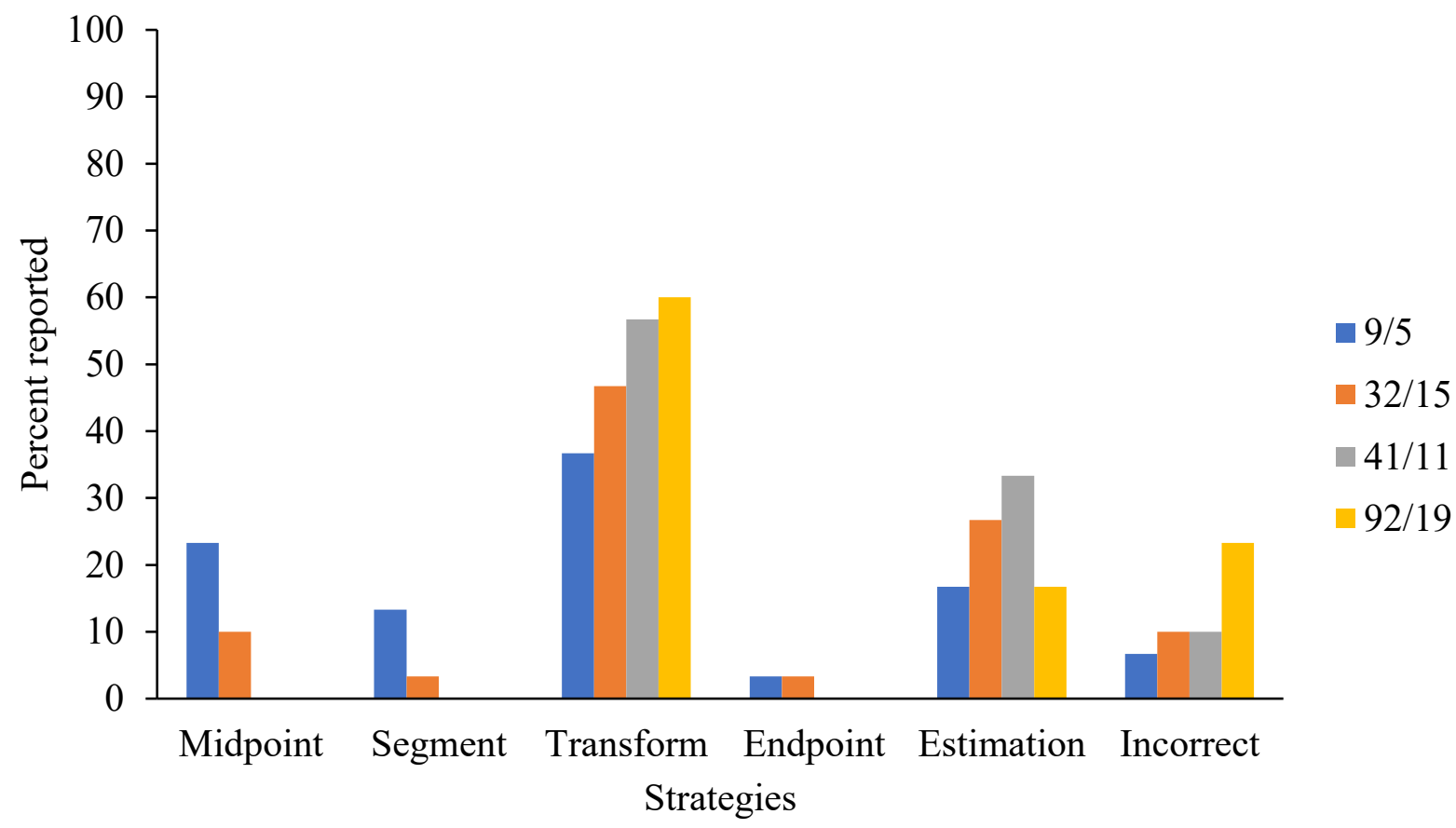

The reports for fraction 32/15 and 41/11 were almost identical. Participants reported transforming the fractions to divisions, that is, 30/15 (46\%) and 44/11 (57\%) respectively, and used these transformations as reference points to place their estimates. For 32/15, strategy use varied, $X^{2}(5, N=30)=26, p<.001$. A variety of strategies were reported, including using the midpoint (10\%), estimating the value of the fraction (27\%), endpoint reference (3\%), segmenting the line (3\%), and incorrect strategies (i.e., "I don't know" (3\%), "around 1.2" (3\%), and "15 is half of 30 went around the middle of the line" $(3 \%))$. For 41/11, strategy use varied, $X^{2}(5, N=$ $30)=43.1, p<.001$. Estimation was the second most frequent strategy $(33 \%)$, with the remaining participants using an incorrect strategy $(10 \%)$.

Finally, the fraction 92/19 was difficult for participants, as shown by more frequent errors in strategy use on this fraction than on the others. Strategy use varied, $X^{2}(5, N=30)=43.9, p<$ 
$.001 .60 \%$ of participants used a transformation strategy that involved rounding the numerator or denominator, or both the numerator and denominator to an easier fraction to estimate (e.g., $100 / 20,90 / 20,95 / 19) .17 \%$ of participants estimated to the nearest whole number, reporting " 4 point something". The remaining participants (23\%) made incorrect estimates reporting, "19 goes into 927 or 8 times" or made incorrect guesses about the fractions magnitude.

In summary, the most frequent strategy used on the improper fractions was to transform the numbers by rounding them to give an easier fraction to estimate. The next most common approach was to round off the whole fraction to the nearest integer using estimation. The strategies commonly used on the $0-1$ fraction number line (i.e., midpoint and endpoint strategies) were uncommon for these improper fractions.

Mixed Fractions. Mixed numbers were 1 9/16, 2 7/8, 3 5/7, 4 4/9. In contrast to the improper fraction strategies, participants' strategy reports on mixed fractions were more similar to those used on the $0-1$ fraction number line. Participants focused their attention on the fractional remainders rather than on finding the whole number interval. Interestingly, the mixed number lead digit acted as a reference point and thus some participants treated the task as a $0-1$ fraction number line mentioning the midpoint between the whole number intervals as well as the endpoint of the interval acting as the endpoint. The strategy reports are shown in Figure 16 for the four mixed fractions. 


\section{Figure 16}

Strategies Reported on the 0-5 Fraction Number Line for Mixed Numbers

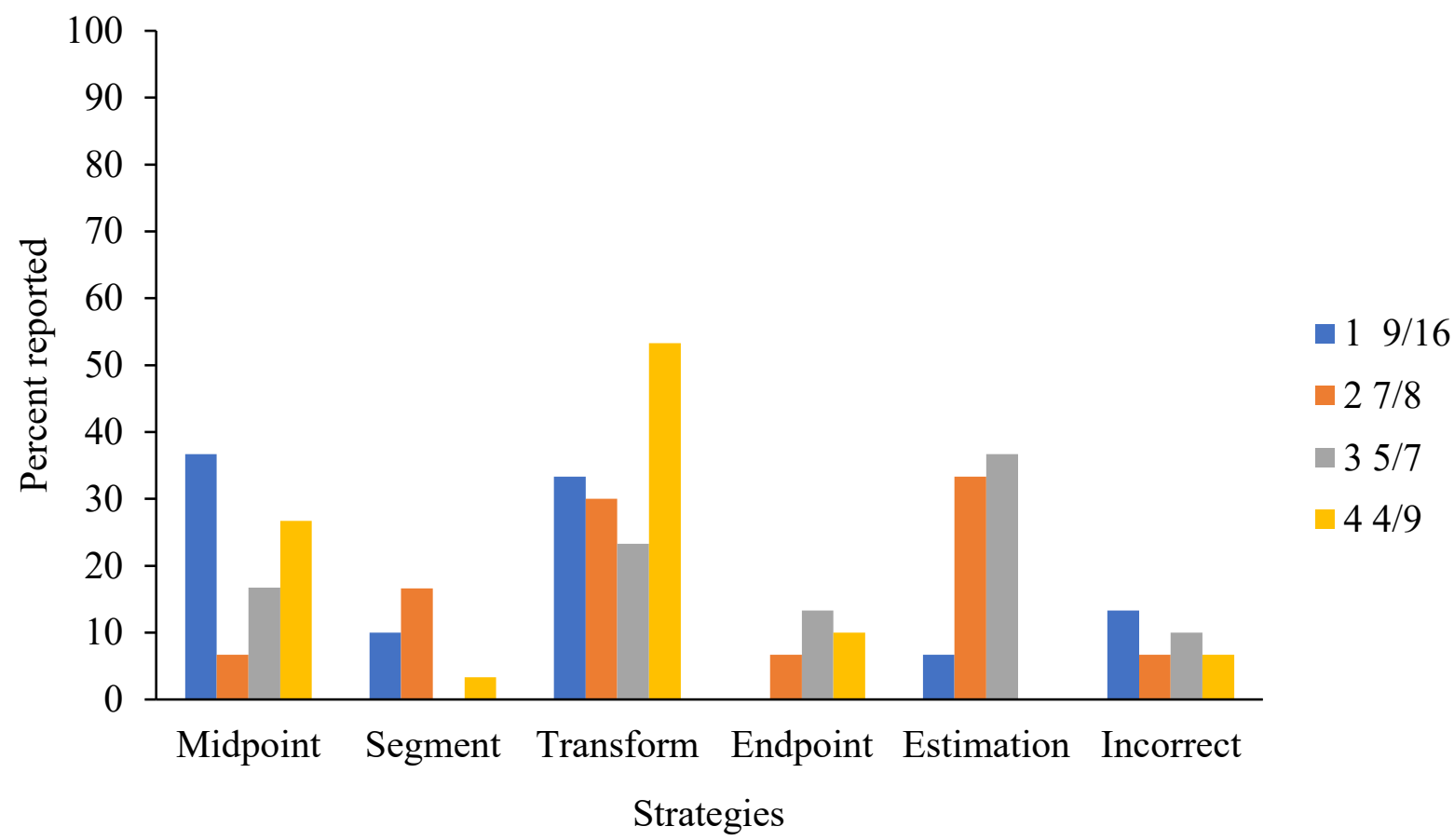

As shown in Figure 15, strategy reports on 1 9/16 and 4/9 were similar presumably because they were both located near the halfway point of their respective whole number intervals (i.e., 1 and 2, 4 and 5 respectively). For this reason, both of these fractions were also easier to transform to a decimal (i.e., approximately 1.5 or 4.5). Thus, midpoint and transformation strategies were more frequent for these fractions than estimation (i.e., identifying the nearest whole number and placing the fraction around there). For $1 \mathbf{9 / 1 6}$, strategy use varied, $X^{2}(5, N=$ $30)=20, p=.001 .17 \%$ of participants identified the midpoint of the interval as $8 / 16$ reporting just above this point while the remaining midpoint references were not specific and just reported "more than half of the interval" (20\%). Transformation strategies for this fraction involved reporting "close to 1.5 ". For the fraction $44 / 9$, strategy use varied, $X^{2}(5, N=30)=13.6, p=$ $.018 .37 \%$ of participants reported transforming the fraction to a decimal "almost 4.5 ". 
Interestingly, $13 \%$ of participants transformed the fraction to an improper fraction (40/9). Participants using a midpoint strategy on this fraction (27\%) were not specific about the midpoint possibly because this was an odd numbered denominator fraction; they reported "less than half of the interval".

For the fraction $\mathbf{2} \mathbf{7 / 8}$, strategy use varied, $X^{2}(5, N=30)=14, p=.016$. The most frequently used strategies were estimation (33\%) and transformation (30\%). For estimation, participants reported "less than 3". This fraction was also the fraction that was most easily used in a segmenting strategy among the mixed numbers as it was the closest to its whole number interval. $30 \%$ of participants reported a transformation strategy on $27 / 8$. Of these, $17 \%$ transformed the fraction to the improper fraction $23 / 8$, which allowed the participants to reinterpret/estimate the fraction as 24/8. It was easier for these participants to look at the fraction as whole numbers that were being divided then as separate pieces (i.e., lead digit and fractional remainder). Another $13 \%$ transformed the fraction to a decimal ( 2.8 or 2.9$) .7 \%$ of participants used the endpoint of the whole number interval to place their estimates, reporting " 2 and $7 / 8$ is almost a whole". Finally, for the fraction $3 \mathbf{5} / 7$, strategy use varied, $X^{2}(5, N=30)=36.8, p<$ .001 . The most frequent strategy was estimation (37\%). $10 \%$ of participants transformed the fraction to an improper fraction. More participants used an endpoint reference for this fraction compared to others using 5 to anchor the estimates closer to the end of the line.

Summary. In summary, upon reviewing strategies used for both improper and mixed numbers there are some differences that stand out. First, transformation is more frequently used to transform fractions from improper to mixed number than mixed number to improper. Second, although segmenting was the most reported strategy for the 0-1 fraction number line, it was much less frequently reported for the $0-5$ fraction number line. It is likely that participants were 
in fact segmenting but were more focused on processing the complex fractions than on the actual placement on the number line, and thus failed to report the segmenting portion of their placement. It is also possible that instead of segmenting for every fraction, participants segmented the line into 5 once at the start and made their estimates from those implicit reference points. 


\section{Discussion}

Fractions are a challenging topic across development, but they are central to mathematics learning because competence with fractions predicts later math achievement, for example, in algebra (Bailey et al., 2012). Number line estimation is an important tool to help students with reasoning about fraction magnitudes and the ability to place fractions on a number line is a goal of the Ontario curriculum for students in grades 4 to 6 (Ministry of Education, 2005). Thus, understanding children's and adults' strategies on fraction number line tasks can inform our understanding about what kinds of information a student needs to complete this task and which strategies allow for better accuracy. Adults and children use multiple strategies in computations, computational estimation, fraction magnitude comparisons, and number line estimation (Fazio et al., 2016; Lemaire \& Lecacheur, 2002; Siegler, Thompson, and Schneider, 2011; Xu et al., 2014). This variability in strategy use is adaptive as it produces accurate and quick responses based on problem features and participant characteristics (Fazio et al., 2016; Lemaire \& Lecacheur, 2002; Siegler, 1989). I sought to investigate whether strategy use is also adaptive in fraction number line estimation with students in grade 4 , grade 6 , and first-year university.

This study provides three main interesting findings about strategy reports on fraction number line estimation. First, strategy flexibility was found to be advantageous but only for adults and only on the $0-1$ fraction number line. Grade 4 and grade 6 students were not advanced enough in their fraction understanding to demonstrate the benefits of multiple strategies.

Moreover, the difficulty of the 0-5 fraction number line challenged the extent to which adults were able to estimate fraction magnitudes. In turn, the complexity of fractions and participants' lack of familiarity with these fractions presumably lead to a reduction in strategies available. When fraction formats require participants to use more steps to understand a fraction's 
magnitude, fewer resources are allotted to choosing a correct strategy for the placement of the fraction on the number line. The resources are instead directed to understanding the fraction's magnitude and not the best strategy to use to accurately place that magnitude on a number line.

Second, when comparing performance across fractions on the $0-1$ fraction number line, adults performed similarly across all fractions whereas children were less accurate on the fractions in the middle of the number line. This finding was supported by strategy report analyses, such that children were more likely to use correct endpoint strategies than correct midpoint strategies. In contrast, there was no difference in implementation between strategies for adults. Adults made few strategy errors compared to children and strategy type did not have an effect on implementation errors.

Third, I replicated previous findings that adults and children make more accurate estimates on integer number lines than on fraction number lines (e.g., Fazio et al, 2014, 2016), presumably because the complexity of the number line increases from the $0-1000$ number line to the $0-1$ fraction number line to the $0-5$ fraction number line due to features of the target number. Finally, on the 0-5 fraction number line, fraction format (i.e., mixed vs. improper and double digit vs. single digit) did not affect estimation accuracy, however, format did affect response times. Specifically, improper fractions with double-digit denominators took longer to estimate than those in the other categories. The finding that fraction format only affected response times and not estimation accuracy suggest that participants transform fractions between formats, however, transformation is effortful.

In summary, the results of this research indicate that, although fractions are used in daily life and are important for students' progress in math education, fractions remain separate from whole numbers in that fractions are less familiar and more challenging. The current study 
showed that even among recent high school graduates, fractions are actively avoided in calculations and problem solving (i.e., shown in the completion of the fraction knowledge assessment). Many participants had forgotten how to complete fraction arithmetic procedures even though they self-reported that their last math course had been less than a year ago. In this sample, students needed to use active processing to place fractions on a number line and participants responded more slowly on fraction number lines than on the integer number line. Thus, there are individual differences in student's familiarity and confidence in dealing with fractions.

Moreover, the analyses of strategy flexibility demonstrated that there is a point in fraction development, between grade 6 and first-year university, in which participants have gained enough conceptual fraction knowledge to have multiple strategies available to them. This transition in fraction development allows strategy flexibility to become important for improved accuracy and response times on fraction number line estimation tasks. Strategy report results suggest that there are some differences between the way children and adults complete this task. With children, internal benchmarks may not be as accurate as they are in adults with experience and practice. Even though using the midpoint is one of the relatively easier strategies, children in grade 4 and 6 struggle estimating the position of fractions close to this benchmark. In contrast, adults make successful estimates around the midpoint and show a pattern of estimating similarly across fractions and strategy use.

\section{Limitations}

This study has some limitations that are worth discussion. First, a major limitation of this study was the small sample size for adults. The sample size made it difficult to interpret correlations as well as make sound conclusions from the data. My original plan called for 60 
participants, but I had to stop collecting data due to the COVID pandemic. Despite the smaller sample size, the depth of strategy analyses into adults' individual strategy use adds strength to conclusions drawn. Second, another limitation of this study was not being able to have a $4^{\text {th }}$ group made of teenagers to add to the age comparisons. A group of students in grade 8 or in high school would have rounded out the study and made comparisons to adults more interesting. These students could have been tested on the more difficult measures that adults were tested on and may have changed the extent to which adults were relative experts.

Third, a more specific limitation was with respect to the $0-5$ fraction number line. This task had a large number of stimuli, which although helped balance the stimuli types was also taxing to the participants. I tried to limit the extent of which participants would be tired by having 2 blocks of stimuli. However, even with a break between blocks, the number of trials was a lot for participants to handle. Testing participants on three types of number lines with similar stimuli becomes repetitive. It is possible that because of the length of the 0-5 fraction number line task, participants were less careful in placing their estimates, which might explain the range of percent accuracy errors. Overall, the testing process was very fraction heavy, and this is difficult for participants who are not in math related fields.

Finally, although I defined strategy flexibility as the number of strategies used, the construct of strategy flexibility is quite complex. The simple definition of strategy flexibility that I chose may have limited the extent to which I could capture participants' knowledge of strategies. Moreover, the way I conceptualized strategy flexibility did not capture efficient strategy use or whether participants chose the optimal strategy for each fraction. For these reasons, I believe that asking participants more detailed questions about what different strategies 
could be used to complete this task might have provided some useful information about participants' knowledge of strategies.

\section{Future Research}

In future studies, researchers should examine the extent to which stimulus type affects performance. For example, double-digit denominators might influence performance on the $0-1$ fraction number line more than the $0-5$ fraction number line because there is more space to be precise. On the 0-5 fraction number line, participants often rounded fractional remainders which did not greatly affect the estimate. In contrast, however on the $0-1$ fraction number line, the fraction needs to be processed completely, which may lead to an effect of number of digits in the denominator. Data from older children would also be useful in investigating whether or not strategy flexibility affects performance on various fraction number lines. Data from experts in math may help to identify whether more experience with fractions results in similar performance between integer and fraction number lines. Moreover, analyses of expert performance could help to determine whether accurate strategy use or use of multiple different strategies is the most efficient approach.

\section{Conclusion}

The present study is important for three reasons. One, it highlights the need for people to have flexibility in the later stages of fraction development and understanding. Two, it captures performance differences across grades that are supported by strategy analyses. Three, in attempting to challenge adults and push the extent of their knowledge of fraction magnitudes and proportional reasoning on a fraction number line task, I found that they were equipped to deal with fractions in various formats (i.e., mixed vs. improper). The implications of this study are that fractions are less familiar and more challenging to estimate than whole numbers, but for 
adults, all fractions are equally challenging, and that using more strategies may facilitate reasoning about fraction magnitudes with adults. 


\section{References}

Ashcraft, M., \& Moore, A. (2012). Cognitive processes of numerical estimation in children. Journal of Experimental Child Psychology, 111(2), 246-267. https://doi.org/10.1016/j.jecp.2011.08.005

Bailey, D., Hoard, M., Nugent, L., \& Geary, D. (2012). Competence with fractions predicts gains in mathematics achievement. Journal of experimental child psychology, 113, 447-455. 10.1016/j.jecp.2012.06.004.

Behr, M., \& Post, T.(1992). Teaching rational number and decimal concepts: research based methods. Teaching mathematics in Grades K-8: Research Based Methods. 2nd Sub Edition, 201-248.

Bonato, M., Fabbri, S., Umiltà, C., \& Zorzi, M. (2007). The mental representation of numerical fractions: Real or integer? Journal of Experimental Psychology: Human Perception and Performance, 33(6), 1410-1419. https://doi.org/10.1037/0096-1523.33.6.1410

Bugden, S., \& Ansari, D. (2011). Individual differences in children's mathematical competence are related to the intentional but not automatic processing of Arabic numerals. Cognition, 118(1), 32-44. https://doi.org/10.1016/j.cognition.2010.09.005

Connolly, A. J. (2008). KeyMath- 3 Diagnostic Assessment, Pearson.

Crooks \& Alibali (2017) Defining and measuring conceptual knowledge in mathematics. Developmental Review, 34, 344-377. http://dx.doi.org/10.1016/j.dr.2014.10.001

Davidson, A. (2012). Making it in America. The Atlantic, 65-83. http://www.theatlantic.com/magazine/archive/2012/01/making-it-in-america/308844/ 
DeWolf, M., Grounds, M., Bassok, M., \& Holyoak, K. (2014). Magnitude comparison with different types of rational numbers. Journal of Experimental Psychology. Human Perception and Performance, 40(1), 71-82.

DeWolf, M., \& Vosniadou, S. (2015). The representation of fraction magnitudes and the whole number bias reconsidered. Learning and Instruction, 37. 10.1016/j.learninstruc.2014.07.002.

Di Lonardo, S., Huebner, M., Newman, K., \& LeFevre, J.-A. (2019). Fixated in unfamiliar territory: Mapping estimates across typical and atypical number lines. Quarterly Journal of Experimental Psychology, 73(2). https://doiorg.proxy.library.carleton.ca/10.1177/1747021819881631

Fazio, L., Bailey, D., Thompson, C., \& Siegler, R. (2014). Relations of different types of numerical magnitude representations to each other and to mathematics achievement. Journal of Experimental Child Psychology, 123, 53-72. https://doi.org/10.1016/j.jecp.2014.01.013

Fazio, L., \& Dewolf, M., \& Siegler, R. (2016). Strategy Use and Strategy Choice in Fraction Magnitude Comparison. Journal of experimental psychology. Learning, memory, and cognition, 42. 10.1037/xlm0000153.

Fazio, L. K., \& Siegler, R. S. (2011). Teaching fractions. In Educational practices series, 22, 28. Geneva: International Academy of Education International Bureau of Education.

Feigenson, L., Dehaene, S., \& Spelke, E. (2014). Core systems of number. TRENDS in Cognitive Science, 8(7). 
Fitzsimmons, C., Thompson, C., \& Sidney, P. (2020). Do adults treat equivalent fractions equally? Adults strategies and errors during fraction reasoning. Journal of Experimental Psychology. Learning, Memory, and Cognition. DOI:10.1037/xlm0000839

Fuchs, L., Schumacher, R., Long, J., Namkung, J., Hamlett, C., Cirino, P., Jordan, N., Siegler, R., Gersten, R., \& Changas, P. (2013). Improving at-risk learners' understanding of fractions. Journal of Educational Psychology.

Hallet, D., Nunes, T., \& Bryant, P. (2010). Individual differences in conceptual and procedural knowledge when learning fractions. Journal of Educational Psychology, 102(2), 395406.

Hallet, D., Nunes, T., Bryant, P., \& Thorpe, C. (2012). Individual differences in conceptual and procedural fraction understanding: The role of abilities and school experience. Journal of Experimental Child Psychology, 113(4), 469-486. https://doi.org/10.1016/j.jecp.2012.07.009

Hamdan, N., \& Gunderson, E. (2017). The number line is a critical spatial-numerical representation: Evidence from a fraction intervention. Developmental psychology, 53(3), 587-596. 10.1037/dev0000252.

Hannula, M. (2003). Locating Fractions on a Number Line. In N.A. Pateman, B.J. Dougherty and J.Zilliox (eds), Proceedings of the 2003 Joint Meeting of the PME and PMENA, International Group for the Psychology of Mathematics Education, 3, 17-24.

Hansen, N., Jordan, N. C., Fernandez, E., Siegler, R. S., Fuchs, L., Gersten, R., \& Micklos, D. (2015). General and math-specific predictors of sixth-graders' knowledge of fractions. Cognitive Development, 35, 34-49.

Hume, T., \& Hume, S. (2014). EstimationLine App. 
Jump Math (2011). Toronto JUMP Math.

Kullberg, A. (2010) What is taught and what is learned. Professional insights gained and shared by teachers of mathematics. Goteborg: Acta Universitatis Gothoburgensis. DOI: 10.13140/RG.2.2.17823.76967

Lortie-Forgues, H., Tian, J., \& Siegler, R. (2015). Why is learning fraction and decimal arithmetic so difficult? Developmental Review, 38, 201-221. 10.1016/j.dr.2015.07.008.

Lemaire, P., \& Lecacheur, M. (2002). Children's strategies in computational estimation. Journal of Experimental Child Psychology, 82(4), 281-304. https://doi.org/10.1016/S0022$\underline{0965(02) 00107-8}$

Lemaire, P., \& Redder, L. (1999). What affects strategy selection in arithmetic? The example of parity and five effects on product verification. Memory \& Cognition, 27(2), 264.382. https://doi.org/10.3758/BF03211420

Martin, W. G., Strutchens, M. E., \& Elliott, P. C. (2007). The learning of mathematics. Reston, VA: National Council of Teachers of Mathematics, 69.

McCloskey, M. (2007). Quantitative literacy and developmental dyscalculias. In D. B. Berch \& M. M. M. Mazzocco (Eds.), Why is math so hard for some children? The nature and origins of mathematical learning difficulties and disabilities, 415-429. Baltimore, MD: Paul H. Brookes Publishing.

Meert, G., Grégoire, J., Seron, X., \& Noel, M. P. (2011). The mental representation of the magnitude of symbolic and nonsymbolic ratios in adults. Quarterly journal of experimental psychology, 65, 702-724. 10.1080/17470218.2011.632485. 
Namkung, J., \& Fuchs, L. (2016). Cognitive predictors of calculations and number line estimation with whole numbers and fractions among at risk students. Journal of Educational Psychology, 108(2), 214-228.

National Mathematics Advisory Panel (NMAP). (2008). Foundations for success: The final report of the National Mathematics Advisory Panel. Washington, DC: Department of Education. http://www2.ed.gov/about/bdscomm/list/mathpanel/ index.html

Ni, Y., \& Zhou, Y. (2005) Teaching and learning fraction and rational numbers: the origins and implications of whole number bias. Educational Psychologist, 40(1), 27-52. DOI: 10.1207/s15326985ep4001_3

Noparit, T., \& Saengpun, J. (2013). How student teachers use proportional number line to teach multiplication and division of fraction: professional learning in context of lesson study and open approach. Creative Education, 4, 19-24. 10.4236/ce.2013.48A005.

Novillis-Larson, C. (1980). Locating proper fractions on number lines: Effect of length and equivalence. School of science and mathematics, 53(5), 423-428.

Ministry of Education (2005). The Ontario curriculum, grades 1-8 Mathematics. Toronto: Ontario, Ministry of Education.

Ontario (1997). EQAO: Education Quality and Accountability Office. Toronto: The Office. Pearson (2003). Math makes sense 8. Toronto: Pearson/Addison Wesley.

Peeters, D., Degrande, T., Ebersbach, M., Verschaffel, L., \& Luwel, K. (2015). Children's use of number line estimation strategies. European Journal of Psychology of Education, 31(2), $117-134$. 
Peeters, D., Sekeris, E., Verschaffel, L., \& Luwel, K. (2017). Evaluating the effect of labeled benchmarks on children's number lin estimation performance and strategy use. Frontiers in Psychology, 8. https://doi.org/10.3389/fpsyg.2017.01082

Resnick, I., Jordan, N., Hansen, N., Rajan, V., Rodrigues, J., Siegler, R.S., Fuchs, L.S. (2016). Developmental growth trajectories in understanding of fraction magnitude from fourth through sixth grade. Developmental psychology, 52(5), 746-746.

Reys, B., Kim, O.-K., \& Bay, J. (1999). Take time for action: establishing fraction benchmarks . Mathematics Teaching in the Middle School, 4(8), 530-532.

Rinne, L. F., Ye, A., Jordan, N. C. (2017). Development of fraction comparison strategies: a latent transition analysis. Developmental Psychology, 53(4), 713-730. http://dx.doi.org/10.1037/dev0000275

Rittle-Johnson, B., Siegler, R. S., \& Alibali, M. W. (2001). Developing conceptual understanding and procedural skill in mathematics: an iterative process. Journal of Educational Psychology, 93(2), 346-62.

https://proxy.library.carleton.ca/login?url=https://search-proquestcom.proxy.library.carleton.ca/docview/62274269?accountid=9894

Schneider, M., Merz, J., Sticker, J., De Smedt, B., Torbeyns, J., Verschaffel, L., et al. (2018). Associations of number line estimation with mathematical competence: a metaanalysis. Child Development, 89, 1467-1484. doi: 10.1111/cdev.13068

Schneider, M., Siegler, R. (2010) Representations of the magnitudes of fractions. Journal of Experimental Psychology: Human Perception and Performance, 36(5), 1227-1238. https://doi.org/10.1037/a0018170 
Sidney, P. G., Thalluri, R., Buerke, M. L., \& Thompson, C. A. (2019) Who uses more strategies? Linking mathematics anxiety to adults' strategy variability and performance on fraction magnitude tasks, Thinking \& Reasoning, 25(1), 94-131.

DOI:10.1080/13546783.2018.1475303

Siegler, R. (1989). Strategy diversity and cognitive assessment. Psychological Science, Educational Researcher, 18(9), 15-20. https://doi.org/10.3102/0013189X018009015

Siegler, R., \& Booth, J. (2008). Numerical magnitude representations influence arithmetic learning. Child Development, 79(4), 1016-1031.

Siegler, R., Carpenter, T., Fennell, F., Geary, D., Lewis, J., Okamoto, Y. et al. (2010). Developing effective fractions instruction for kindergarten through 8th grade: A practice guide (NCEE \#2010-4039), Washington, DC: National Center for Education Evaluation and Regional Assistance, Institute of Education Sciences, U.S. Department of Education.

Siegler, R., Duncan, G., Davis-Kean, P., Claessens, A., Engel, M., Susperreguy, M. I., \& Chen, M. (2012). Early predictors of high school mathematics achievement. Psychological science. 23, 691-697. 10.1177/0956797612440101.

Siegler, R., \& Lortie-Forgues, H. (2015). Conceptual knowledge of fraction arithmetic. Journal of Educational Psychology, 107(3), 909-918. https://doi.org/10.1037/edu0000025

Siegler, R., \& Opfer, J. (2003). The development of numerical estimation: Evidence of multiple representations of numerical quantity. Psychological science, 13(3), 237- 243. https://doi.org/10.1111/1467-9280.02438

Siegler, R., \& Pyke, A. (2013). Development and individual differences in understanding of fractions. Developmental Psychology, 49(10), 1994-2004. doi: $\underline{10.1037 / a 0031200}$ 
Siegler, R., Thompson, C., \& Schneider, M. (2011). An integrated theory of whole number and fractions development. Cognitive psychology. 62, 273-296.

10.1016/j.cogpsych.2011.03.001.

Slusser, E., Santiago, R., \& Barth, H. (2012). Developmental change in numerical estimation. Journal of Experimental Psychology: General, 142(1), 193-208. https://doi.org/10.1037/a0028560

Sowinski, C., Dunbar, K., \& LeFevre, J.-A. (2014). Calculation fluency test. Math Lab, Carleton.

Stigler, J., Givvin, K., \& Thompson, B. (2010). What community college developmental mathematics students understand about mathematics. MathAMATYC Educator.

Vamvakoussi, X., \& Vosniadou, S. (2004). Understanding the structure of the set of rational numbers: a conceptual change approach. Learning and Instruction, 14(5), 453-467.

Witherspoon, T. (2019). Fifth graders' understanding of fractions on the number line. School Science and Mathematics, 119(6), 340-352. https://doi.org/10.1111/ssm.12358

Xu, C., \& LeFevre, J.-A. (2016). Training young children on sequential relations among numbers and spatial decomposition: Differential transfer to number line and mental transformation tasks. Developmental Psychology, 52(6), 854-866. https://doi.org/10.1037/dev0000124

Xu, C., LeFevre, J.-A., Wells, ., \& Imbo, . (2014). Strategy flexibility in computational estimation for Chinese- and Canadian- educated adults. Journal of Experimental Psychology Psychology: Learning, Memory, and Cognition, 40(5), 1481-1497. https://doi.org/10.1037/a0037346 
Zhang, D., Stecker, P., \& Beqiri, K. (2017). Strategies students with and without mathematics disabilities use when estimating fractions on number lines. Learning Disability Quarterly, 40(4), 225-236. https://doi.org/10.1177/0731948717704966. 


\section{Appendices}

Appendix A: Full List of Tasks Completed by Children

\begin{tabular}{|c|c|c|c|c|}
\hline Measure & Classification & $\begin{array}{l}\text { Time } \\
(\mathrm{min})\end{array}$ & S1 & S2 \\
\hline Parent Survey & & 15 & & \\
\hline \multicolumn{5}{|l|}{ Cognitive Skills } \\
\hline Spatial span ${ }^{a}$ & Spatial attention & 3 & $\checkmark$ & \\
\hline Digit span forward ${ }^{b}$ & Short term memory & 2 & & $\checkmark$ \\
\hline Digit span backward ${ }^{b}$ & Working memory & 2 & $\checkmark$ & \\
\hline \multicolumn{5}{|l|}{ Language Skills } \\
\hline PPVT - English ${ }^{c}$ & Vocabulary & 5 & $\checkmark$ & \\
\hline WIAT & Reading & 2 & $\checkmark$ & \\
\hline \multicolumn{5}{|l|}{ Basic Symbolic Math Skills } \\
\hline Number comparison ${ }^{\mathrm{a}}$ & Basic quantity & 3 & & $\checkmark$ \\
\hline Order judgement ${ }^{a}$ & Basic ordinal & 3 & & $\checkmark$ \\
\hline Symbol decision task ${ }^{\mathrm{a}}$ & Symbolic knowledge & 3 & $\checkmark$ & \\
\hline 0-1000 number line ${ }^{a}$ & Ordinal and proportional & 3 & $\checkmark$ & \\
\hline \multicolumn{5}{|l|}{$\begin{array}{l}\text { Linking Language and Math } \\
\text { Abilities }\end{array}$} \\
\hline Math words ${ }^{b}$ & Math vocabulary & 5 & & $\checkmark$ \\
\hline Transcoding ${ }^{b, c}$ & Language/ digit mapping & 5 & $\checkmark$ & \\
\hline \multicolumn{5}{|c|}{ Outcomes: general math abilities } \\
\hline Key Math Problem Solving ${ }^{\mathrm{b}, \mathrm{c}}$ & & 5 & $\checkmark$ & \\
\hline Key Math Algebra ${ }^{b}$ & & 5 & & $\checkmark$ \\
\hline Arithmetic Fluencyc & & 3 & & $\checkmark$ \\
\hline Fraction Mapping ${ }^{a}$ & & 3 & $\checkmark$ & \\
\hline Fraction Word Problems ${ }^{b}$ & & 5 & & $\checkmark$ \\
\hline Fraction Number-line ${ }^{a}$ & & 4 & & $\checkmark$ \\
\hline Total testing time & & & 31 & 30 \\
\hline
\end{tabular}

$\mathrm{S} 1=$ session 1

$\mathrm{S} 2=\operatorname{session} 2$ 
Notes: a) iPad based tasks b) Oral tasks c) Paper-based tasks 
Participant ID code

How old are you?

$\nabla 15$ or younger (1) ... 60 or older (46)

Gender

Male (1)

Female (2)

Other (3)

What program are you currently in?

BA (1)

BSc (2)

BEng (3)

BEd (4)

BComm (5)

BCompSci (6)

BPAPM (7)

MA (8)

MSc (9)

Other (please specify): (15)

Year in program:

What is your current major?

V Psychology (1) ... Other (17)

If other please specify: 
In which country did you attend ELEMENTARY school?

Canada (1)

Iran (2)

China (and/or Hong Kong) (3)

Nigeria (4)

United Arab Emirates (5)

Taiwan (6)

Another Country (please specify): (7)

In which country did you attend HIGH SCHOOL?

Canada (1)

Iran (2)

China (and/or Hong Kong) (3)

Nigeria (4)

United Arab Emirates (5)

Taiwan (6)

Another Country (please specify): (7)

What was the last grade (or equivalent course level) in which you took a mathematics course?

Grade 9 (1)

Grade 10 (2)

Grade 11 (3)

Grade 12 (4)

Grade 13 (5)

Other (Please Specify): (6)

What languages do you speak?

English (1)

French (2)

Chinese/Mandarin/Cantonese (3)

Farsi (4)

Arabic (5)

Igbo (6) 
Swahili (7)

Creole (8)

Hindi (9)

Other (please specify): (10)

What do you consider your first language

English (1)

French (2)

Chinese/Mandarin/Cantonese (3)

Farsi (4)

Arabic (5)

Igbo (6)

Swahili (7)

Creole (8)

Hindi (9)

Other (please specify): (10)

Please rate your level of skill in these areas of mathematics:

\begin{tabular}{|c|c|c|c|c|c|}
\hline & Very Low (1) & $(2)$ & Moderate (3) & (4) & Very High (5) \\
\hline $\begin{array}{c}\text { Basic } \\
\text { mathematical } \\
\text { skill (e.g. } \\
\text { arithmetic } \\
\text { skill) (1) }\end{array}$ & & & & & \\
\hline $\begin{array}{l}\text { More complex } \\
\text { areas of } \\
\text { mathematics } \\
\text { (e.g. calculus, } \\
\text { algebra) (2) }\end{array}$ & 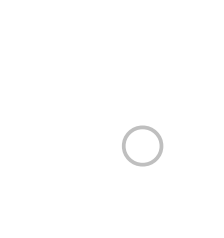 & & & & \\
\hline
\end{tabular}

How often do you avoid situations involving mathematics?

\section{Almost Always}

(1)

(2) Sometimes (3)
(4)

Almost Never

(5)

I avoid them:

(1)

How often do you find that situations involving mathematics make you nervous?

Almost Always

(1)
(2)

Sometimes (3)
(4)

Almost Never

(5)

They make me nervous: (1) 
How difficult was mathematics for you in high school?

Extremely

Difficult (1)
(2)

Math was: (1)
Moderately

Difficult (3)

(4)

Not At All

Difficult (5)

You have now completed the survey. Please tell the experimenter you are done. Thank you for your participation! 


\section{Appendix C: Full List of Digit Backwards Stimuli}

\begin{tabular}{|c|c|c|c|}
\hline Item & Score $(0 / 1)$ & Span 2 & Score $(0 / 1)$ \\
\hline 1. & $2-1$ & $1-3$ & \\
\hline 2. & $3-5$ & $6-4$ & \\
\hline 3. & $5-7-4$ & $2-5-9$ & \\
\hline 4. & $7-2-9-6$ & $8-4-9-3$ & \\
\hline 5. & $4-1-3-5-7$ & $9-7-8-5-2$ & \\
\hline 6. & $1-6-5-2-9-8$ & $3-6-7-1-9-4$ & \\
\hline 7. & $8-5-9-2-3-4-6$ & $4-5-7-9-2-8-1$ & \\
\hline 8. & $6-9-1-7-3-2-5-8$ & $3-1-7-9-5-4-8-2$ & \\
\hline
\end{tabular}


Appendix D: Fraction Knowledge Assessment

\section{Fraction Knowledge Assessment}

\section{Carleton \\ U N I V E R S I T Y \\ Canada's Capital University}

Please answer the following questions to the best of your ability. You may use pencil and paper in front of you to do your work. These questions will be timed, so try to complete them as quickly and as accurately as possible. Once you click next, the timer will start! Any questions?

$$
\frac{4}{5}-\frac{5}{8}=
$$

Please solve the problem above:

$$
4 \frac{2}{5}+3 \frac{3}{4}=
$$

Please solve the problem above:

Mary and John both have pocket money. Mary spends $1 / 4$ of hers, while John spends $1 / 2$ of his.

a) Is it possible for Mary to have spent more than John?

b) Why do you think so? 


\section{$\frac{3}{5} \times \frac{5}{12}=$}

Please solve the problem above:

$\frac{2}{3} \div \frac{2}{6}=$

Please solve the problem above:

Estimate the sum of $\frac{12}{13}+\frac{7}{8}$.

Which of the following numbers is the closest to this answer?

1

2

19

21 


$$
1-\frac{5}{12}=
$$

Please solve the problem above:

$$
\begin{array}{llll}
\frac{3}{4} & \frac{2}{5} & \frac{5}{4} & \frac{2}{3}
\end{array}
$$

Arrange these fractions in order of size, from the smallest on the top to the largest on the bottom.

$2 / 5$

$2 / 3$

$3 / 4$

$5 / 4$

How many possible fractions are there between $1 / 4$ and $1 / 2$ ?

$$
2 \times \frac{1}{8}=
$$

Please solve the problem above:

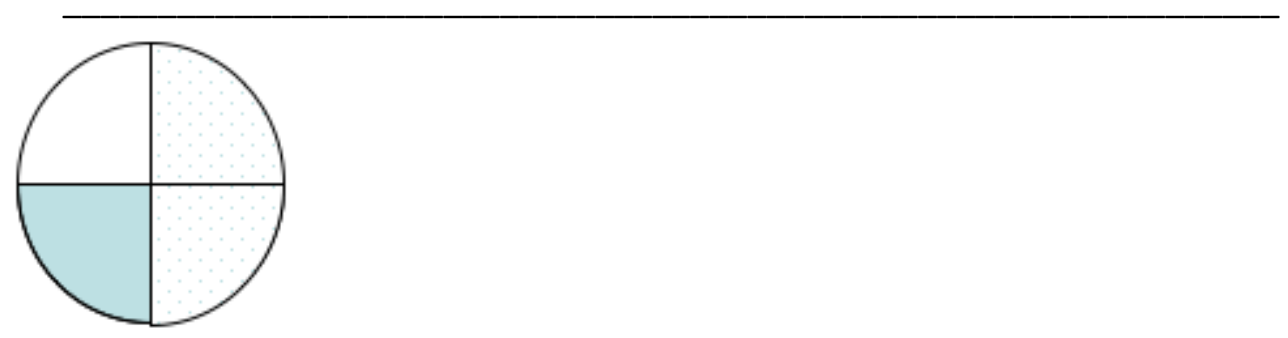

If I shaded in $1 / 6$ of the checkered part of the disc, what fraction of the whole disc would I have shaded?

$1 \frac{1}{5}-\frac{3}{5}=$ 
Please solve the problem above:

A relay race is run in stages of $1 / 8 \mathrm{~km}$ each. Each runner runs one stage. How many runners would be required to run a total distance of $3 / 4 \mathrm{~km}$ ?

$$
\frac{3}{4} \div \frac{3}{8}=
$$

Please solve the problem above:

Thank you for taking this Survey! 